\title{
Irregular conformal block, spectral curve and flow equations
}

\author{
Sang Kwan Choi, Chaiho Rim and Hong Zhang \\ Department of Physics and Center for Quantum Spacetime (CQUeST), \\ Sogang University, \\ 35 Baekbeom-ro, Mapo-gu, Seoul 0410\%, Korea \\ E-mail: hermit1231@sogang.ac.kr, rimpine@sogang.ac.kr, \\ kilar@sogang.ac.kr
}

ABSTRACT: Irregular conformal block is motivated by the Argyres-Douglas type of $\mathrm{N}=2$ super conformal gauge theory. We investigate the classical/NS limit of irregular conformal block using the spectral curve on a Riemann surface with irregular punctures, which is equivalent to the loop equation of irregular matrix model. The spectral curve is reduced to the second order (Virasoro symmetry, $\mathrm{SU}(2)$ for the gauge theory) and third order $\left(W_{3}\right.$ symmetry, SU(3)) differential equations of a polynomial with finite degree. The conformal and $\mathrm{W}$ symmetry generate the flow equations in the spectral curve and determine the irregular conformal block, hence the partition function of the Argyres-Douglas theory ala AGT conjecture.

KEYwords: Conformal and W Symmetry, M(atrix) Theories, Nonperturbative Effects

ARXIV EPRINT: 1510.09060 


\section{Contents}

1 Introduction 1

2 Irregular conformal block with Virasoso symmetry 3

2.1 Irregular matrix model and spectral curve 3

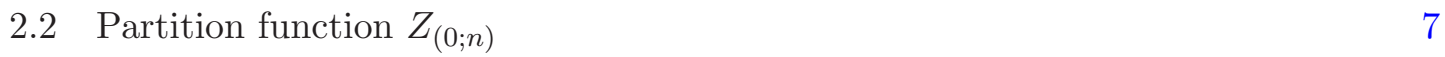

2.3 Partition function $Z_{(m ; n)}$ and ICB 9

3 Irregular conformal block with $W_{3}$ symmetry 12

$\begin{array}{lll}3.1 & \text { Irregular matrix model and loop equation } & 12\end{array}$

$\begin{array}{ll}3.2 & \text { Spectral curve and partition function } \\ 3.3 & \text { Partition fun }\end{array}$

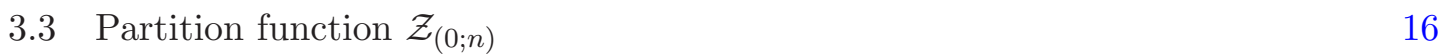

$\begin{array}{ll}3.4 & \text { Partition function } \mathcal{Z}_{(m ; n)} \text { and ICB } \\ \end{array}$

4 Relation with gauge theories $\quad 19$

5 Conclusion and outlook $\quad 22$

$\begin{array}{ll}\text { A Loop equation of } A_{2} \text { irregular matrix model } & 23\end{array}$

B Representation of W3 currents $\quad 26$

$\begin{array}{ll}\text { C Perturbation method to find flow equations in } A_{2} \text { model } & 27\end{array}$

$\begin{array}{lll}\text { C.1 } & \mathcal{Z}_{(0 ; 1)} & 28\end{array}$

$\begin{array}{lll}\text { C.2 } & \mathcal{Z}_{(0 ; 2)} & 31\end{array}$

$\begin{array}{lll}\text { C.3 } & \mathcal{Z}_{(1 ; 1)} & 33\end{array}$

\section{Introduction}

Irregular conformal block (ICB) is closely related with Argyres-Douglas type (AD) of N=2 super conformal gauge theory in four dimensions [1]. AD type has the non-trivial infrared fixed point on the Coulomb branch and does not allow marginal deformation. Therefore, AD type of gauge theory is considered as a special class of super conformal gauge theory.

According to AGT [2], the Nekrasov partition function [3-5] of the gauge theory is equivalent to the conformal block of Liouville vertex operators in two dimensions. This connection is understood using the twisted compactification of the six dimensional $\mathcal{N}=$ $(2,0)$ theory on a punctured Riemann surface [6-8]. In this context, the Seiberg-Witten curve of the four-dimensional theory is identified with the spectral curve of Hitchin system on the Riemann surface with regular punctures. The Hitchin system has simple poles and the residues are associated with the mass parameter of the gauge theory [9, 10]. On the 
other hand, the $\mathrm{AD}$ type theory is characterized in terms of irregular punctures, poles of higher order $[11,12]$. Therefore, the irregular puncture is the key point to understand AD type theory.

It is noted that irregular punctures appear when the regular conformal block has the colliding limit $[13,14]$. The colliding limit is a fusion of vertex operators so that multiple moments of Liouville charges of the vertex operators are present. It is like the collection of charges distributed over a small region whose collection is viewed as an idealized system of total charge, dipole, quadrupole and multi-poles. As a consequence, the irregular punctures maintain the conformal symmetry but change the conformal state. Note that a regular puncture on the Riemann surface appears due to a primary field, which indicates the primary state, eigen-state of Virasoro generator $L_{0}$. Similarly, the irregular puncture of order $(n+1)$ indicates an irregular state of rank $n$, which is a simultaneous eigen-state of positive Virasoro generators $L_{k}$ with $n \leq k \leq 2 n$. This irregular state is called a Gaiotto state [15] or a Whittaker state [16]. Rank 0 state corresponds to the regular state.

According to AGT, the conformal block provides the partition function of gauge theory. In the same way, the partition function of AD type gauge theory is given by ICB. To find ICB, we will use the property of the Penner-type random matrix model. The Penner-type matrix model is originally introduced to study the topological structure of the punctured Riemann surface [17]. It turns out that the Liouville conformal block is conveniently represented as the Penner-type matrix model, which is an equivalent way of writing of the Selberg integrals $[18,19]$.

There are a few merits in using the matrix model. The Penner-type matrix model is easy to apply the colliding limit which results in the irregular matrix model (IMM). From IMM one may obtain ICB by properly normalizing the partition function and compensating $\mathrm{U}(1)$ factor [20]. In addition, the random matrix model provides the loop equation, Wardidentity representing the conformal symmetry. The loop equation allows one to investigate the detailed structure of the spectral curve corresponding to the Hitchin system. It turns out that the spectral curve contains flow equations corresponding to the conformal and $\mathrm{W}$ symmetry and fixes ICB and therefore, the partition function of AD type gauge theory according to AGT. It is remarkable that one may find ICB using only the conformal and W symmetry of the theory. This paper is mainly devoted to elaborating on the relation between ICB and spectral curve through flow equations.

This paper is organized as follows. In section 2, we consider the case with $\mathrm{su}(2)$ gauge group which has the Virasoro symmetry. We confine ourselves to the classical/NS (Nekrasov-Shatashivili) limit [21] to present the main features of the system. NS limit is a limit where one of the deformation parameters $\epsilon_{1,2}$ of the guage theory goes to zero and the other (called $\epsilon$ ) remains to be finite. In the Liouville theory sense, the classical limit is achieved by taking the limit $\epsilon=\hbar Q$ finite where the scale parameter $\hbar$ goes to zero but the background charge $Q$ goes to infinite. One may resort to the classical/NS limit to get simplified spectral curve which still possesses the irregular punctures and the Virasoro symmetry. This spectral curve reduces to the second order differential equation of a polynomial of a finite degree $N$, size of the random matrix. In addition, we do not have the cut structure on the Riemann surface which usually appears at the large $N$ limit. 
However, the concept of the filling fraction (identified with the Coulomb branch parameter in the gauge theory) still remains and plays an important role in ICB. We present the explicit form of the flow equations from the spectral curve and use the flow equations to find the the irregular partition function and ICB. An explicit form of the irregular partition function and ICB are given for lower rank cases.

In section 3 we extend the idea to the case with su(3) gauge group, which has the Virasoro and $W_{3}$ symmetry. IMM of $A_{2}$ is presented, which is obtained from the Toda field theory and its colliding limit. The spectral curve is obtained and flow equations are found by identifying the Virasoro and $W_{3}$ symmetry generator. Using a similar method, we construct partition function of IMM and ICB. In section 4, we briefly summarize the relation between gauge theories and the matrix model approach. Section 5 is the conclusion and outlook. In the appendix, one can find the derivation of the loop equations of $A_{2}$ model (appendix A), representation of $W_{3}$ currents (appendix B), and perturbative method to find the moments for the flow equation (appendix $\mathrm{C}$ ).

\section{Irregular conformal block with Virasoso symmetry}

\subsection{Irregular matrix model and spectral curve}

The irregular matrix model with Virasoro symmetry is given as the $\beta$ deformed random matrix model

$$
Z_{(m ; n)}=\int\left(\prod_{I=1}^{N} d \lambda_{I}\right) \prod_{I<J}\left(\lambda_{I}-\lambda_{J}\right)^{2 \beta} e^{\frac{\sqrt{\beta}}{g} \sum_{I} V\left(\lambda_{I}\right)},
$$

whose potential has the form

$$
V(z)=c_{0} \log z-\sum_{k=1}^{n}\left(\frac{c_{k}}{k z^{k}}\right)+\sum_{\ell=1}^{m}\left(\frac{c_{-\ell} z^{\ell}}{\ell}\right) .
$$

The deformed parameter $\beta$ is related with the Liouville screening charge $b=i \sqrt{\beta}$. In addition, a small expansion parameter $g$ is introduced in the partition function, which is equivalent to the Liouville scaling parameter $\hbar=-2 i g ; \sqrt{\beta} / g=-2 b / \hbar$. It is noted that the parameter $c_{k}$ in the potential is the one denoted as $\hat{c}_{k}$ in [22]. We simplify the notation by deleting hat and therefore, $c_{k}$ stands for old $\hbar c_{k}$.

The partition function (2.1) is obtained from the colliding limit of regular $(m+n+2)$ point conformal block. As a result, the potential parameter $c_{k}$ is related with the Liouville charge $\alpha_{a}$ and the vertex operator position $z_{a}$ as $c_{k}=\sum_{r=1}^{n} \hbar \alpha_{r}\left(z_{r}\right)^{k}$ with $k>0$ and $c_{-\ell}=-\sum_{a=1}^{m} \hbar \alpha_{a}\left(z_{a}\right)^{-\ell}$ with $\ell>0$. One may regard the partition function as the irregular correlation of two irregular vertex operators of rank $m$ and $n$, considering the rank- $n$ irregular operator as $I_{c}^{(n)}(z)=e^{2 \Phi(z)}$ with $\Phi(z)=\sum_{k=0}^{n} c_{k} \partial_{k} \varphi / k$ ! where $\partial_{k} \varphi$ stands for $k$ th derivative of the Liouville field. In this sense, the rank-0 irregular operator reduces to the regular vertex operator $V_{\alpha}(z)=e^{2 \alpha \varphi(z)}$. In addition, the two -point irregular correlation is viewed as the inner product of irregular states of rank $m$ and $n$. Indeed, the two-point correlation is identified with the inner product if one takes care of the proper normalization 
of the state and $\mathrm{U}(1)$ factor which arises at the colliding limit [20]. Multi-point irregular conformal block is also constructed in a straight-forward way.

The partition function has the conformal symmetry and the symmetric property is encoded in the loop equation,

$$
4 W(z)^{2}+4 V^{\prime}(z) W(z)+2 \epsilon W^{\prime}(z)=f(z) .
$$

Here $W(z)$ is the resolvent, $W(z)=g \sqrt{\beta}\left\langle\sum_{I} \frac{1}{z-\lambda_{I}}\right\rangle$ where the bracket $\langle\cdots\rangle$ denotes the expectation value with respect to the matrix model. $f(z)=4 g \sqrt{\beta}\left\langle\sum_{I} \frac{V^{\prime}(z)-V^{\prime}\left(\lambda_{I}\right)}{z-\lambda_{I}}\right\rangle$. The loop equation (2.3) is given at the classical/NS limit. Note that the classical/NS limit is obtained when $\hbar \rightarrow 0$ and $b \rightarrow \infty$ so that $\epsilon=\hbar b$ is finite and multi-point resolvent contribution vanishes [23]. Therefore, we ignore the two-point resolvent in the original loop equation.

From the Liouville field theory point of view, the large $b$ limit is the same as the one with the small $b$ limit because of the duality $b \rightarrow 1 / b$ is present in of the Liouville theory. ${ }^{1}$ Therefore, the same finite $\epsilon=\hbar Q$ is obtained either at the NS limit or at the classical limit $(b \rightarrow 0)$ of the Liouville theory. It is also noted that the same duality also appears in the corresponding gauge theory. The $\Omega$ deformation parameters $\epsilon_{1}$ and $\epsilon_{2}$ are identified as $\epsilon_{1}=\hbar b$ and $\epsilon_{2}=\hbar / b$ according to to AGT conjecture and $\epsilon=\epsilon_{1}+\epsilon_{2}$. The $b \rightarrow 1 / b$ duality corresponds to $\epsilon_{1} \rightarrow \epsilon_{2}$ duality. If one of the deformation parameter vanishes while the other remains finite, the limit is called Nekrasov-Shatashivili (NS) limit, which is the same as the classical limit of the Liouville theory or the matrix model.

The loop equation (2.3) can be put in a more informative form if one uses $x=2 W+V^{\prime}$,

$$
x^{2}+\epsilon x^{\prime}+\xi_{2}(z)=0,
$$

which can be regarded as a spectral curve with $z$ the (complex) spectral parameter. The analytic structure of the spectral curve is specified by $\xi_{2}(z)=-V^{\prime 2}+\epsilon V^{\prime \prime}-f$ which has a pole of order $2 n$ and a zero of order $2 m$ on the Riemann surface:

$$
\xi_{2}(z)=\sum_{k=-2 m}^{2 n} \frac{\Lambda_{k}}{z^{k+2}}-\sum_{a=-m}^{n-1} \frac{d_{a}}{z^{a+2}},
$$

where $f=\sum_{a=-m}^{n-1} \frac{d_{a}}{z^{a+2}}$ is used. The spectral curve indicates that one may view the mode of $\xi_{2}(z)$ as the conserved quantity appearing in the integrable theories $[9,10]$. Indeed, if one ignores $f(z)$ (putting $\left.d_{a}=0\right), \xi_{2}(z)$ is given in terms of $\Lambda_{k}$ which is a constant:

$$
\Lambda_{k}=\epsilon(k+1) c_{k}-\sum_{r+s=k} c_{r} c_{s}
$$

The new feature in the loop equation $(2.4)$ is that $f(z)$ has the special role in finding the partition function [22]. This role is closely related with the Virasoro symmetry. Note

\footnotetext{
${ }^{1}$ Note that before taking the classical/NS limit the parameter $\epsilon$ in the loop equation is $\hbar Q=2 g(\sqrt{\beta}-$ $1 / \sqrt{\beta}$ ) where $Q=b+1 / b$ is the background charge of the Liouville theory. Thus, the original $\epsilon$ is invariant under the $b \rightarrow 1 / b$ duality.
} 
that $\xi_{2}(z)$ is identified with the expectation value of the energy momentum tensor (Virasoro current)

$$
\xi_{2}(z)=\langle T(z)\rangle=\sum_{k \in \mathbb{Z}} \frac{\left\langle L_{k}\right\rangle}{z^{k+2}} .
$$

Comparing the two, one notes that $\Lambda_{k}$ is the eigenvalue of the Virasoro mode $L_{k}$ for $n \leq k \leq 2 n$ and $-2 m \leq k \leq-m$. The positive mode $L_{k}$ with $n \leq k \leq 2 n$ applies on the ket so that the ket is the simultaneous eigenstate of $L_{k}(n \leq k \leq 2 n)$. The negative mode applies on the bra since $L_{k}^{\dagger}=L_{-k}$ and therefore, the bra is the simultaneous eigenstate of $L_{k}(-2 m \leq k \leq-m)$.

However, the extra mode $\Lambda_{a}+d_{a}(-m \leq a \leq n-1)$ present in $\xi_{2}(z)$ is not a simple constant due to $d_{a}$ and therefore, not an eigenvalue. Instead, the mode represents the expectation value of $L_{a}$. It is noted that $d_{a}$ is directly related to the partition function or free energy $F_{(m ; n)}=-\hbar^{2} \log Z_{(m ; n)}[24]$ :

$$
d_{a}= \begin{cases}v_{a}\left(F_{(m ; n)}\right) & \text { if } 0 \leq k \leq n-1 \\ v_{a}\left(F_{(m ; n)}\right)+2 \epsilon N c_{k} & \text { if }-m<a<0 \\ 2 \epsilon N c_{-m} & \text { if } a=-m,\end{cases}
$$

where $v_{a}$ is given as derivative with respect to the parameters $c_{k}$ 's:

$$
v_{a}= \begin{cases}\sum_{s>0} s\left(c_{s+a} \frac{\partial}{\partial c_{s}}\right) & \text { if } 0 \leq k \leq n-1 \\ \sum_{s<0}(-s)\left(c_{s+a} \frac{\partial}{\partial c_{s}}\right) & \text { if }-m<a<0 .\end{cases}
$$

In fact, $v_{a}$ in $(2.9)$ is the representation of Virasoro mode $L_{a}$ on the parameter space and $d_{a}$ is the vector flow $v_{a}$ of the partition function. It is, however, noted that $v_{a}$ behaves differently, depending on the sign of $a$. When $a \geq 0, v_{a}$ acts on the parameter space $\left\{c_{1}, \cdots, c_{n}\right\}$ of the right state and satisfies the commutation relation $\left[v_{k}, v_{\ell}\right]=-(k-\ell) v_{k+\ell}$. On the other hand, $v_{a}$ with $a<0$ acts on the parameter space $\left\{c_{-m}, \cdots, c_{-1}\right\}$ of the left state and satisfies the commutation relation $\left[v_{k}, v_{\ell}\right]=(k-\ell) v_{k+\ell}$.

The appearance of left/right representation of $L_{k}$ brings an unpleasant feature. Suppose we evaluate $Z_{(0 ; 1)}$ whose parameter space is $\left\{c_{1}\right\}$. The vector flow $d_{0}$ of $v_{0}$ can determine the partition function using (2.8). In fact, the partition function is easily obtained if one scales $\lambda_{I} \rightarrow c_{1} \lambda_{I}$ in (2.1). This shows that the flow equation (2.8) contains the information of the parameter scaling. On the other hand, suppose one evaluates $Z_{(1: 0)}$ whose parameter space is $\left\{c_{-1}\right\}$. The partition function is found if one scales $\lambda_{I} \rightarrow \lambda_{I} / c_{-1}$. However, there is no $v_{-1}$ representation in (2.8) when $m=1$ and we cannot fix the partition function using the flow equation. This unpleasant feature does not raise any problem in evaluating $Z_{(m ; n)}(m \neq 0)$ if one scales $\lambda_{I}^{m} \rightarrow \lambda_{I}^{m} / c_{-m}$ first. (See more details in subsection 2.2.)

Nevertheless, this unpleasant feature can be avoided if one takes into account of the conformal invariance of the partition function. Note that conformal transformation $\lambda_{I} \rightarrow$ $1 / \lambda_{I}$ changes the parameter space $\left\{c_{-m}, \cdots, c_{-1}, c_{1}, \cdots, c_{n} ; c_{0}\right\}$ into its dual space 
$\left\{\bar{c}_{-n}, \cdots, \bar{c}_{-1}, \bar{c}_{1}, \cdots, \bar{c}_{m} ; \bar{c}_{0}\right\}$ where $\bar{c}_{k}=-c_{-k}(k \neq 0)$ and $\bar{c}_{0}=c_{\infty}$. Here $c_{\infty}$ is determined by the neutrality condition

$$
c_{0}+c_{\infty}+N \epsilon=\epsilon .
$$

The neutrality condition is hidden in the matrix model but is manifest in the Liouville conformal block. The conformal block is evaluated using the perturbation with the insertion of $N$ screening operators and the neutrality condition is required $\sum_{a} \alpha_{a}+N b=Q$ so that the conformal block is non-vanishing. At the colliding limit, the neutrality condition reduces to $c_{0}+c_{\infty}+N \hbar b=\hbar Q$ where $c_{0}=\sum_{r=1}^{n} \hbar \alpha_{r}$ is the total Liouville charge (scaled by $\hbar)$ at the origin and $c_{\infty}$ is the charge at infinity. If one has the classical/NS limit, one has the neutrality condition (2.10).

Using this conformal symmetry, we have two left representations instead of one left and one right representations: one is the original representation $v_{a}$ with $a \geq 0$ which applies to original parameter space $\left\{c_{1}, \cdots, c_{n}\right\}$ with parameters $\left\{c_{-m}, \cdots, c_{-1} ; c_{0}\right\}$ intact. The other is $\bar{v}_{a}$ with $a \geq 0$ which applies to the dual parameter space $\left\{\bar{c}_{-n}, \cdots, \bar{c}_{-1}\right\}$ with $\left\{\bar{c}_{1}, \cdots, \bar{c}_{m} ; \bar{c}_{0}\right\}$ intact. Here the barred representation $\bar{v}_{a}$ is defined in the same form as in (2.9) but with $\left\{c_{k}\right\}$ replaced with $\left\{\bar{c}_{k}\right\}$ and commutes with $v_{a} ;\left[v_{a}, \bar{v}_{b}\right]=0$. The barred partition function is the same as the original one because of the conformal invariance of the partition function. Therefore, one may find the partition function from the flow equation presented in the spectral curve and the existence of the partition function is guaranteed due to the consistency condition of $d_{a}$ and its dual $\bar{d}_{a}$ :

$$
\begin{aligned}
& v_{a}\left(d_{b}\right)-v_{b}\left(d_{a}\right)=-(a-b) d_{a+b} \\
& \bar{v}_{a}\left(\bar{d}_{b}\right)-\bar{v}_{b}\left(\bar{d}_{a}\right)=-(a-b) \bar{d}_{a+b} \\
& v_{a}\left(\bar{d}_{b}\right)-\bar{v}_{b}\left(d_{a}\right)=0
\end{aligned}
$$

It is simple to note that if one replaces $d_{a}$ with $\Lambda_{k}$, then $\Lambda_{k}$ also satisfies the consistency condition (2.11).

If one uses the flow equation to construct the partition function, the major step is to find the values of $d_{a}$ and $\bar{d}_{a}$ directly from the analytic property of the spectral curve (2.4). In the usual large $N$ approach [25, 26], one expands the spectral curve in powers of $\hbar$ keeping $\hbar N=O(1)$ and assuming $x$ is $O(1)$. In this case, $\hbar Q$ is the sub-dominant order of $\hbar$ [22] and the spectral curve is given as $x= \pm \sqrt{-\xi_{2}}$ at the leading order. The solution results in $(m+n)$ square-root branch cuts and provides the double covering of the Riemann surface $[18,27]$. The contour integral of $x$ over a certain cut becomes an elliptic integral and is identified with the filling fraction. In this way, one can find $d_{k}$ in terms of the filling fraction and the parameters $c_{k}$ 's. Once $d_{k}$ is known, the free energy is constructed according to (2.8) and (2.9). This procedure is very interesting from the gauge theory point of view. Since the filling fraction is identified with the Coulomb branch parameter of the gauge theory according to AGT and the spectral curve is the Seiberg-Witten curve of the Hitchin system, Seiberg-Witten curve determines the partition function of the ArgyresDouglas gauge theory with the parameters $\left\{c_{k}\right\}$ which can be rewritten in terms of masses and Coulomb branch expectation value of the gauge theory. 
At the classical/NS limit, the procedure does not change much. Still, there appear important modifications. There are no branch cuts in the spectral curve: only $N$-number of simple poles are present. This fact can be seen as follows. Let us consider an expectation value

$$
P(z) \equiv\left\langle\prod_{I}\left(z-\lambda_{I}\right)\right\rangle=\sum_{A=0}^{N} P_{A} z^{A}=\prod_{\alpha}\left(z-z_{\alpha}\right)
$$

which is a polynomial of degree $N$ with $P_{N}=1 . z_{\alpha}$ 's are $N$-zeros of the polynomial. We assume all zeros are distinct. Note that $P(z)$ is related with the resolvent $W(z)$ at the classical/NS limit since

$$
\log \left(\frac{P(z)}{P\left(z_{0}\right)}\right)=\frac{2}{\epsilon} \int_{z_{0}}^{z} d z^{\prime} W\left(z^{\prime}\right) .
$$

Here we use the fact the multi-point resolvent contribution vanishes at the classical/NS limit $[23,28]$. Taking the derivative of $(2.13)$, one has $2 W(z)=\epsilon P^{\prime}(z) / P(z)$ which can be put as

$$
2 W(z)=\epsilon \sum_{\alpha=1}^{N} \frac{1}{z-z_{\alpha}} .
$$

Therefore, only $N$-simple poles appear in the spectral curve, which substitute the cuts present at the large $N$ expansion.

The monic polynomial $P(z)$ has the central role in finding $d_{a}$. Explicitly, the spectral curve (2.4) reduces to the second order differential equation of $P(z)$ : from (2.3) one has

$$
\epsilon^{2} P^{\prime \prime}(z)+2 \epsilon V^{\prime}(z) P^{\prime}(z)=f(z) P(z) .
$$

One may expand the differential equation in power series of $z$ and find finite number of algebraic relations of $P_{A}$ 's and $d_{a}$ 's since $P(z)$ is the polynomial of degree $N$. Therefore, one can find $d_{a}$ 's using the algebraic relations only. It should be noted that the solution is not unique. The solution depends on how the zeros of the polynomial $P(z)$ distribute around the stationary point of the potential. Therefore, the filling fraction may be applied to the distribution of the zeros even though there are no branch cuts on the Riemann surface. With the filling fraction one may fix the relevant solution of (2.15). We provide some explicit examples in the next two subsections.

\subsection{Partition function $Z_{(0 ; n)}$}

In this section we first consider the case where the potential is given as logarithmic and inverse polynomials only: $c_{k}=0$ when $k<0$. The partition function with this potential is regarded as two point correlation between one regular and one irregular vertex operator. In this case, $d_{0}$ is simply obtained if one uses large $z$ expansion of the loop equation:

$$
d_{0}=2 \epsilon c_{0} N+\epsilon^{2} N(N-1) .
$$

However, other values of $d_{k}$ are not easy to find.

Suppose one evaluates the simplest partition function, rank $n=1$. In this case, $d_{0}$ is enough to find the partition function if one uses the flow equation $(2.8) . Z_{(0 ; 1)}=$ 
$c_{1}^{-d_{0} / \hbar^{2}} \mathcal{N}_{(0 ; 1)}$ where $\mathcal{N}_{(0 ; 1)}$ is the normalization independent of $c_{1}$. The same result is obtained if one rescales $\lambda_{I}$ in the partition function (2.1). In addition, one can find $P(z)$ explicitly since the mode expansion gives a recursive relation: $P_{A}=\xi_{A+1} P_{A+1}$ where $\xi_{A+1}=2(A+1) \epsilon c_{1} /\left(d_{0}-2 \epsilon c_{0} A-\epsilon^{2} A(A-1)\right)$. Here $P_{N+1}=0$ and $\xi_{N+1}=0$ are used for notational simplicity and $P_{N}=1$. The recursion relation shows that for $0 \leq A<N$

$$
P_{A}=\prod_{\alpha=A+1}^{N} \xi_{\alpha}
$$

Suppose $N=1$, one has $P(z)=z-z_{1}$ and $P_{0}=\xi_{1}=c_{1} / c_{0}=-z_{1}$. The zero $z_{1}$ corresponds to the stationary point of the potential $V$. When $N>1$, the solution $P_{A}$ provides the information of $N$ zeros around the stationary point since $P_{A}$ is written as the the polynomial of $N$-zeros in a permutation invariant form: $P_{A}=\sum_{\left\{\alpha_{i}\right\}} z_{\alpha_{1}} \cdots z_{\alpha_{N-A}}$ where the index sum is ordered.

If the rank is greater than one, the solutions $P_{A}$ and $d_{k}$ are more complicated. To get the idea on this solution, let us consider the rank two with $N=1$. In this case we need $d_{0}$ and $d_{1} . d_{0}$ is trivially given: $d_{0}=2 \epsilon c_{0}$. On the other hand, one has $d_{1}=2 \epsilon c_{1}-d_{0} P_{0}$ and $d_{1}$ is fixed by a quadratic equation:

$$
d_{1}^{2}-2 \epsilon c_{1} d_{1}+2 \epsilon c_{2} d_{0}=0 .
$$

One has two solutions: $d_{1}^{ \pm}=\epsilon c_{1}(1 \pm \sqrt{1-\eta})$ where $\eta=4 c_{2} c_{0} / c_{1}^{2}$. Note that two solutions shows that $P_{0}^{-} \sim c_{1} / c_{0}$ and $P_{0}^{+} \sim c_{2} / c_{1}$, each of which lies near one of two stationary points of the potential. This two different solutions are due to the fact that zeros of the polynomial (or the poles of the resolvent) distribute around two different stationary points of the potential. One may classify the solutions in terms of the filling fraction. Therefore, if one integrates the flow equation (2.8), one has the different free energy depending on the filling fraction,

$$
F_{(0 ; 2)}^{\mp}=\epsilon c_{0}\left(\log c_{2}-\frac{1}{8} \int^{\eta} \frac{1 \mp \sqrt{1-x}}{x^{2}} d x\right) .
$$

For general $N$, one can easily convince that $P_{N-1}$ has $N+1$ solutions. The solutions correspond to the zero distribution of $P(z)$ so that $N=N_{1}+N_{2}$ where $N_{1}$ zeros at one stationary point and $N_{2}$ at the other. However, it is not easy to find the exact form of $d_{a}$ when $N$ is large and one may resort to perturbative expansion. One way to find the solution is using the power series of $\epsilon$ with $\epsilon$ small. Note that $W(z)=O(\epsilon)$ and $f(z)=O(1)$ while $V(z)=O(1)$ from the definition. In this case, we may apply the $\epsilon$ expansion to the loop equation (2.3) directly. Denoting $W(z)=\sum_{k \geq 1} \epsilon^{k} W^{(k)}(z)$ and $f(z)=\sum_{k \geq 1} \epsilon^{k} f^{(k)}(z)$, one has the leading order contribution

$$
2 W^{(1)}(z)=\frac{f^{(1)}}{2 V^{\prime}} .
$$

This shows that the poles of the resolvent is located closely to the stationary points of the potential at the leading order. This is consistent with the expectation that zeros of the 
polynomial $P(z)$ are accumulated around the stationary points. Denoting $N_{k}$ for the filling fraction around the stationary point $\xi_{k}$ of the potential, one obtains the identity

$$
N_{k}=\oint_{\mathcal{A}_{k}} \frac{f^{(1)}}{2 V^{\prime}} d z
$$

where $\mathcal{A}_{k}$ is the contour encircling $\xi_{k}$ only. Since the number of stationary point is the same as the number of $d_{a}$, one can fix $d_{a}$ at $O\left(\epsilon^{1}\right)$. At the second order of the loop equation, one has the identity

$$
0=\oint_{\mathcal{A}_{k}} d z\left\{\frac{f^{(2)}}{2 V^{\prime}}-\frac{\left(f^{(1)}\right)^{\prime}}{4\left(V^{\prime}\right)^{2}}+\frac{\left(2 V^{\prime \prime}-f^{(1)}\right) f^{(1)}}{8\left(V^{\prime}\right)^{3}}\right\}
$$

Here the identity assumes that the contour $\mathcal{A}_{k}$ encircles all the poles of the resolvent corresponding to the fractional number $N_{k}$. In this way, one finds $d_{a}$ order by order in $\epsilon$ and therefore, the partition function.

For the case rank 2 we have $d_{1}$ :

$$
\begin{aligned}
d_{1}= & -2 \epsilon c_{0}\left(N_{2} \xi_{1}+N_{1} \xi_{2}\right) \\
& +\epsilon^{2} \frac{\xi_{1} \xi_{2}}{\left(\xi_{1}-\xi_{2}\right)^{2}}\left[N_{2}\left(N_{2}-1\right)\left(2 \xi_{1}-\xi_{2}\right)-N_{1}\left(N_{1}-1\right)\left(\xi_{1}-2 \xi_{2}\right)-2 N_{1} N_{2}\left(\xi_{1}+\xi_{2}\right)\right]+\mathcal{O}\left(\epsilon^{3}\right)
\end{aligned}
$$

where $\xi_{1}, \xi_{2}$ are two stationary points satisfying $c_{0} z^{2}+c_{1} z+c_{2}=c_{0}\left(z-\xi_{1}\right)\left(z-\xi_{2}\right)$. Using the flow equations $d_{0}=v_{0}\left(F_{(0: 2)}\right)$ and $d_{1}=v_{1}\left(F_{(0: 2)}\right)$, we get the free energy $F_{(0: 2)}$. The partition function is given up to $\mathcal{O}\left(\epsilon^{2}\right)$,

$$
\begin{aligned}
Z_{(0 ; 2)}= & c_{2}^{-\frac{\epsilon}{\hbar^{2}} c_{0}\left(N_{1}+N_{2}\right)-\frac{\epsilon^{2}}{\hbar^{2}} \frac{3}{4}\left(N_{1}\left(N_{1}-1\right)+N_{2}\left(N_{2}-1\right)\right)}\left(c_{1}^{2}-4 c_{0} c_{2}\right)^{\frac{\epsilon^{2}}{4 \hbar^{2}}\left(N_{1}\left(N_{1}-1\right)+N_{2}\left(N_{2}-1\right)-4 N_{1} N_{2}\right)} \\
& \times\left(\frac{c_{1}+\sqrt{c_{1}^{2}-4 c_{0} c_{2}}}{c_{1}-\sqrt{c_{1}^{2}-4 c_{0} c_{2}}}\right)^{-\frac{\epsilon}{\hbar^{2}} c_{0}\left(N_{1}-N_{2}\right)-\frac{3}{4} \frac{\epsilon^{2}}{\hbar^{2}}\left(N_{1}\left(N_{1}-1\right)+N_{2}\left(N_{2}-1\right)\right)} \\
& \times e^{-\frac{\epsilon}{\hbar^{2}} \frac{c 1}{2 c_{2}}\left(\sqrt{c_{1}^{2}-4 c_{0} c_{2}}\left(N_{2}-N_{1}\right)+c_{1}\left(N_{1}+N_{2}\right)\right)}
\end{aligned}
$$

For the rank $n$ with $N$ zeros, one has $\frac{(N+n-1) !}{N !(n-1) !}$ solutions. One may view this solutions as the zero distribution with $N=\sum_{i=1}^{n} N_{i}$ with $N_{i}$ zeros around each stationary point of the potential. Considering this zero distribution, one may expect that the solutions can be obtained perturbatively using parameter ratio set $\left\{\frac{c_{1}}{c_{0}}, \frac{c_{2}}{c_{1}}, \cdots, \frac{c_{n}}{c_{n-1}}\right\}$. Each ratio stands for each stationary point. The same conclusion also holds for $Z_{(n ; 0)}=\bar{Z}_{(0 ; n)}$ if one works with the barred notations.

\subsection{Partition function $Z_{(m ; n)}$ and ICB}

If one considers the case rank $(m ; n)$, the potential contains finite Laurent series. One may evaluate the flow equations by finding $d_{a}$ 's. The special feature of the partition function 
$Z_{(m ; n)}$ is that one may evaluate ICB of irregular vertex of rank $m$ and $n$. In this case, ICB is identified with the inner product $\left\langle I^{(m)} \mid I^{(n)}\right\rangle$ of two irregular states of rank $m$ and $n$. The relation of ICB with $Z_{(m ; n)}$ is given in [20].

$$
\mathcal{F}_{\Delta}^{(m: n)}\left(\left\{c_{-k}\right\}:\left\{c_{k}\right\}\right)=\frac{e^{\zeta_{(m: n)}} Z_{(m ; n)}\left(c_{0} ;\left\{c_{\ell}\right\}\right)}{\left.Z_{(0: n)}\left(c_{0} ;\left\{c_{k}\right\}\right) Z_{(0: m)}\left(\bar{c}_{0} ;\left\{\bar{c}_{k}\right\}\right)\right)}
$$

$Z_{(m ; n)}$ is divided by $Z_{(0 ; n)}$ and $Z_{(0 ; m)}$ to give the proper normalization. The extra factor $e^{\zeta_{(m: n)}}$ comes when $m$ vertex operators are put at infinity and $n$ operators at the origin. Explicit result is given as $\hbar^{2} \zeta_{(m ; n)}=-\sum_{k}^{\min (m, n)} 2\left(c_{k} c_{-k}\right) / k$. Therefore, it is obvious that ICB is exponentiated and the exponent should be inversely proportional to $\hbar^{2}$ [29], considering the $\hbar$ dependence of the free energies and $\zeta_{(m ; n)}$.

For example, ICB $\mathcal{F}_{\Delta}^{(1 ; 1)}$ is obtained directly using the relation $(2.25) . Z_{(0 ; 1)}\left(c_{0} ; c_{1}\right)$ can be easily obtained from the flow equation $v_{0}\left(F_{(0 ; 1)}\right)=d_{0}$ and its dual $Z_{(0 ; 1)}\left(\bar{c}_{0} ; \bar{c}_{1}\right)$ :

$$
Z_{(0 ; 1)} Z_{(0 ; 1)}=c_{-1}^{\left(\epsilon N\left(\epsilon N+2 c_{0}\right)-\epsilon^{2} N\right) / \hbar^{2}} \eta_{0}^{-\left(\epsilon N_{0}\left(\epsilon N_{0}+2 c_{0}\right)-\epsilon^{2} N_{0}\right) / \hbar^{2}}
$$

where $\eta_{0} \equiv c_{1} c_{-1}$. To evaluate $Z_{(1: 1)}$, we first obtain $c_{-1}$ dependence by rescaling $\lambda_{I} \rightarrow$ $\lambda_{I} / c_{-1}: Z_{(1 ; 1)}=c_{-1}^{\left(\epsilon N\left(\epsilon N+2 c_{0}\right)-\epsilon^{2} N\right) / \hbar^{2}} \widetilde{Z}_{(1 ; 1)} . \quad \widetilde{Z}_{(1: 1)}$ is the partition function with the potential $\widetilde{V}(z)=c_{0} \log z+z-\eta_{0} / z$, which is to be evaluated from the flow equation using $d_{0}=v_{0}\left(-\hbar^{2} \log \widetilde{Z}_{(1 ; 1)}\right)$. We use the $\epsilon$ expansion to obtain the parameter dependence of $d_{0}$ :

$$
d_{0}=-2 \epsilon\left(N_{0} \xi_{1}+N_{\infty} \xi_{2}\right)+\epsilon^{2} \frac{N_{0}\left(N_{0}-1\right) \xi_{1}^{2}-4 N_{0} N_{\infty} \xi_{1} \xi_{2}+N_{\infty}\left(N_{\infty}-1\right) \xi_{2}^{2}}{\left(\xi_{1}-\xi_{2}\right)^{2}}+\mathcal{O}\left(\epsilon^{3}\right)
$$

where $\xi_{1}$ and $\xi_{2}$ are stationary points of $\widetilde{V}(z)$. The flow equation shows that

$$
\begin{aligned}
Z_{(1 ; 1)}= & c_{-1}^{\frac{1}{\hbar^{2}}\left(\epsilon N\left(\epsilon N+2 c_{0}\right)-\epsilon^{2} N\right)} \eta_{0}^{-\frac{\epsilon^{2}}{2 \hbar^{2}}\left(N_{0}^{2}-N_{0}+N_{\infty}^{2}-N_{\infty}\right)}\left(\frac{c_{0}+\sqrt{c_{0}^{2}-4 \eta_{0}}}{c_{0}-\sqrt{c_{0}^{2}-4 \eta_{0}}}\right)^{-\frac{\epsilon^{2}}{2 \hbar^{2}}\left(N_{\infty}^{2}-N_{\infty}-N_{0}^{2}+N_{0}\right)} \\
& \times\left(c_{0}-\sqrt{c_{0}^{2}-4 \eta_{0}}\right)^{-\left(2 \epsilon c_{0} N_{0}\right) / \hbar^{2}}\left(c_{0}+\sqrt{c_{0}^{2}-4 \eta_{0}}\right)^{-\left(2 \epsilon c_{0} N_{\infty}\right) / \hbar^{2}} \\
& \times\left(c_{0}^{2}-4 \eta_{0}\right)^{-\frac{\epsilon^{2}}{4 \hbar^{2}}\left(N_{0}^{2}-N_{0}-N_{\infty}^{2}+N_{\infty}+4 N_{0} N_{\infty}\right)} e^{-\frac{2 \epsilon}{\hbar^{2}}\left(N_{0}-N_{\infty}\right) \sqrt{c_{0}^{2}-4 \eta_{0}}},
\end{aligned}
$$

up to $\mathcal{O}\left(\epsilon^{2}\right)$. From the result, one can obtain $\mathcal{F}_{\Delta}^{(1 ; 1)}$. We provide $\mathcal{F}_{\Delta}^{(1: 1)}$ in powers of $\eta_{0}$ and $\epsilon$ :

$$
\begin{aligned}
\mathcal{F}_{\Delta}^{(1 ; 1)}= & {\left[1-2 \frac{\eta_{0}}{\hbar^{2}}+2 \frac{\eta_{0}^{2}}{\hbar^{4}}+\mathcal{O}\left(\eta_{0}^{3}\right)\right]+\epsilon\left[\frac{2\left(N_{0}-N_{\infty}\right)}{c_{0}} \frac{\eta_{0}}{\hbar^{2}}-\frac{\left(4 c_{0}^{2}-\hbar^{2}\right)\left(N_{0}-N_{\infty}\right)}{c_{0}^{3}} \frac{\eta_{0}^{2}}{\hbar^{4}}+\mathcal{O}\left(\eta_{0}^{3}\right)\right] } \\
& -\epsilon^{2}\left[\frac{2\left(N_{0}^{2}-N_{0}-2 N_{0} N_{\infty}\right)}{c_{0}^{2}} \frac{\eta_{0}}{\hbar^{2}}+\left\{\frac{\hbar^{2}\left(7 N_{0}\left(N_{0}-1\right)+N_{\infty}\left(N_{\infty}-1\right)-16 N_{0} N_{\infty}\right)}{2 c_{0}^{4}}\right.\right. \\
& \left.\left.-\frac{2\left(N_{0}\left(3 N_{0}-2\right)+N_{\infty}^{2}-6 N_{0} N_{\infty}\right)}{c_{0}^{2}}\right\} \frac{\eta_{0}^{2}}{\hbar^{4}}+\mathcal{O}\left(\eta_{0}^{3}\right)\right]+\mathcal{O}\left(\epsilon^{3}\right)
\end{aligned}
$$


One may also evaluate ICB directly using the perturbative approach of IMM as noted in [20]. This is because the relation (2.25) shows that one needs $Z_{(m ; n)}$ with proper normalization and compensates by the U(1) factor. For the perturbative approach one may divide the potential matrix with $N$ eigenvalues into the one with $N_{0}$ eigenvalues and the other with $N_{\infty}$ so that $N=N_{0}+N_{\infty}$. The normalization $Z_{(0 ; n)}$ is obtained if one uses $V_{(0: n)}$ instead of $V_{(m: n)}$ with $N_{0}$ eigenvalues in $Z_{(m ; n)}$. Therefore, one may consider the potential $V_{(m ; n)}$ with $N_{0}$ eigenvalues composed of $V_{(0: n)}$ and the rest so that $V_{(0: n)}$ is treated as the reference potential $V_{0}$ and the rest as the perturbative one $\Delta V_{0}$ :

$$
V_{0}=\sum_{I=1}^{N_{0}}\left(c_{0} \log \lambda_{I}-\sum_{k=1}^{n} \frac{c_{k}}{k \lambda_{I}^{k}}\right), \quad \Delta V_{0}=\sum_{I=1}^{N_{0}}\left(\sum_{\ell=1}^{n} \frac{c_{-\ell}}{\ell} \lambda_{I}^{\ell}\right) .
$$

Likewise, the potential $V_{(m ; n)}$ with $N_{\infty}$ eigenvalues composed of $V_{(m ; 0)}$ as the reference potential $V_{\infty}$ and the perturbation $\Delta V_{\infty}$ The partition function may be rewritten using the conformal transformation $\lambda_{J} \rightarrow 1 / \mu_{J}$ so that one can use the dual form

$$
V_{\infty}=\sum_{J=1}^{N_{\infty}}\left(\bar{c}_{0} \log \mu_{J}-\sum_{\ell=1}^{m} \frac{\bar{c}_{\ell}}{\ell \mu_{J}^{\ell}}\right), \quad \Delta V_{\infty}=\sum_{J=1}^{N_{\infty}}\left(\sum_{k=1}^{n} \frac{\bar{c}_{-k}}{k} \mu_{J}^{k}\right) .
$$

Once the normalization is done, the cross term $\prod_{I, J}\left(1-\lambda_{I} \mu_{J}\right)^{2 \beta}$ remains from the Vandermonde determinant. Therefore, the perturbative approach is to evaluate the expectation value $\left\langle\prod_{I, J}\left(1-\lambda_{I} \mu_{J}\right)^{2 \beta} \exp \left(\frac{\sqrt{\beta}}{g}\left(\Delta V_{0}\left(\lambda_{I}\right)+\Delta V_{\infty}\left(\mu_{J}\right)\right)\right)\right\rangle_{0}$ where $\langle\cdots\rangle_{0}$ refers to the expectation value with respect to the reference potential. Practically, one may evaluate the expectation values from the loop equations of the partition functions $Z_{(0: n)}$ and $Z_{(0: m)}$ by using the large $z$ expansion of the resolvents. However, this perturbative approach needs additional identities for multi-point resolvent correlations even at the classical/NS limit $[22,35]$. We have $\mathcal{F}_{\Delta}^{(1 ; 1)}$ at the classical/NS limit from the result obtained in $[20]$ :

$$
\mathcal{F}_{\Delta}^{(1 ; 1)}=1+\left[\frac{\ell_{1} \ell_{-1}}{2 \hbar^{2} \Delta}\right]+\left[\frac{\ell_{1}^{2} \ell_{-1}^{2}\left(2 \Delta-\hbar^{2}\right)}{16 \hbar^{4} \Delta^{3}}+\frac{\left(3 \ell_{1}^{2}-4 \Delta \ell_{2}\right)\left(3 \ell_{-1}^{2}-4 \Delta \ell_{-2}\right)}{16 \hbar^{2} \Delta^{2}\left(4 \Delta+3 \epsilon^{2}\right)}\right]+\mathcal{O}\left(\eta_{0}^{3}\right) .
$$

where $\Delta=\left(c_{0}+\epsilon N_{0}\right)\left(\epsilon-\left(c_{0}+\epsilon N_{0}\right)\right), \ell_{1}=2 c_{1}\left(\epsilon-c_{0}\right)$ and $\ell_{2}=-c_{1}^{2} \cdot \ell_{-1}$ and $\ell_{-2}$ are its duals. It is noted that $\Delta$ and $\ell_{k}$ correspond to the modes appearing in $\xi_{2}(z)$ and can be represented as the expectation values between regular and irregular state: $\Delta=\left\langle\Delta\left|L_{0}\right| I^{(1)}\right\rangle /\left\langle\Delta \mid I^{(1)}\right\rangle$ where $\left\langle\Delta \mid I^{(1)}\right\rangle$ is $Z_{(0: 1)} . \quad \ell_{+k}=\left\langle\Delta\left|L_{+k}\right| I^{(1)}\right\rangle /\left\langle\Delta \mid I^{(1)}\right\rangle, \ell_{-k}=\left\langle I^{(1)}\left|L_{-k}\right| \Delta\right\rangle /\left\langle I^{(1)} \mid \Delta\right\rangle$ for $k=1,2$ and $\left\langle I^{(1)} \mid \Delta\right\rangle$ is $\bar{Z}_{(0: 1)}$. This identification clearly shows that $\mathcal{F}_{\Delta}^{(1 ; 1)}$ satisfies the dual symmetry observed in section 2.1 .

The result (2.32) is given in power of $\eta_{0}=c_{1} c_{-1}$; each squared bracket in (2.32) corresponds to each order of $\eta_{0}$. One can easily check that the squared bracket term is consistent with the one appeared in [37] if one uses the series expansion in $1 / \hbar^{2}$ at each order of $\eta_{0}^{k}$ and ignores higher order than $1 / \hbar^{2 k}$. This shows that $\mathcal{F}_{\Delta}$ in powers of $1 / \hbar^{2}$ should be considered as the formal series. This fact is already suggested by Zamolodchikov 
and Zamolodchikov [38] for the regular conformal block and investigated fully in [29] for the irregular conformal block: at the classical/NS limit, the (ir)-regular conformal block should behave as $\mathcal{F}_{\Delta} \rightarrow \exp \left(-F / \hbar^{2}\right)$ where the free energy $F$ is finite as $\hbar \rightarrow 0$.

\section{Irregular conformal block with $W_{3}$ symmetry}

\subsection{Irregular matrix model and loop equation}

The irregular matrix model with $W_{3}$ symmetry can be derived from Toda field theories at the colliding limit $[30,36]$. The simplest matrix model is obtained from $A_{2}$ Toda theory. The $A_{2}$ irregular matrix model is two matrix model with potential $V_{1}$ and $V_{2}$ :

$$
\mathcal{Z}_{(m ; n)}=\int \prod_{i=1}^{N} \prod_{j=1}^{M} d x_{i} d y_{j} \Delta(x)^{2 \beta} \Delta(y)^{2 \beta} \Delta(x, y)^{-\beta} e^{\frac{\sqrt{\beta}}{g}\left[\sum_{i=1}^{N} V_{1}\left(x_{i}\right)+\sum_{j=1}^{M} V_{2}\left(y_{j}\right)\right]},
$$

where $\Delta(x)=\prod_{i<k}\left(x_{i}-x_{k}\right)$ and $\Delta(x, y)=\prod_{i, j}\left(x_{i}-y_{j}\right)$ are Vandermonde determinants. $\beta$ is the deformed parameter and conveniently put $\sqrt{\beta}=-i b$ as in the Virasoro case. When $\beta=1$, the model reduces to hermitian two-matrix model and the powers of the Vandermonde determinant correspond to the $A_{2}$ Dynkin index. The potential with rank $(m ; n)$ has the explicit form

$$
\begin{aligned}
& V_{1}(z)=b_{0} \log z-\sum_{k=1}^{n} \frac{b_{k}}{k z^{k}}+\sum_{k=1}^{m} \frac{b_{-k} z^{k}}{k}, \\
& V_{2}(z)=a_{0} \log z-\sum_{k=1}^{n} \frac{a_{k}}{k z^{k}}+\sum_{k=1}^{m} \frac{a_{-k} z^{k}}{k} .
\end{aligned}
$$

To obtain the matrix model we work with the primary operator $V_{a}\left(z_{a}\right)=e^{\vec{\alpha}_{a} \cdot \vec{\varphi}\left(z_{a}\right)}$ where Toda field $\vec{\varphi}$ has the orthogonal components $\vec{\varphi}=\varphi_{1} \frac{(1,1,-2)}{\sqrt{6}}+\varphi_{2} \frac{(1,-1,0)}{\sqrt{2}}$ and satisfy the free field correlation $\varphi_{i}(z, \bar{z}) \varphi_{j}(w, \bar{w}) \sim-\delta_{i j} \log |z-w|^{2}$. The conformal block with $n+m+2$ primary operators are considered with $N$ screening operators of the type $e^{b \vec{e}_{1} \cdot \vec{\varphi}\left(x_{i}\right)}$ and $M$ of type $e^{b \vec{e}_{1} \cdot \vec{\varphi}\left(x_{i}\right)}$. Here $\vec{e}_{1}$ and $\vec{e}_{2}$ are two root vectors of $A_{2}$. The conformal block is non-vanishing if the neutrality condition holds [39]:

$$
\vec{\alpha}_{\infty}+\sum_{a} \vec{\alpha}_{a}+b N \vec{e}_{1}+b M \vec{e}_{2}=2 Q \vec{\rho}
$$

where $Q=b+1 / b$ is the background charge and $\vec{\rho}=\vec{e}_{1}+\vec{e}_{2}$ is the Weyl vector.

The correlation between screening operators provides the Vandermonde determinant with powers related to the Dynkin index of the two roots. The potential is obtained if one has the colliding limit of the colliding limit of the primary operators. If the Toda momentum of the primary operator is presented as $\vec{\alpha}_{a}=\alpha_{a} \frac{(1,1,-2)}{\sqrt{3}}+\beta_{a}(1,-1,0)$ and $n+1$ operators are fused to the origin and $m+1$ operators at infinity, one has the potentials in (3.2) with the parameters: $b_{k} \equiv \sum_{a=0}^{n} \hbar \beta_{a} z_{a}^{k}(k=0,1, \cdots, n)$ and $b_{-k} \equiv-\sum_{a=0}^{n} \hbar \beta_{a} z_{a}^{k}$ $(k=1, \cdots, m)$. Similarly, $a_{\ell}=\left(\sqrt{3} c_{\ell}-b_{\ell}\right) / 2$ and $c_{k} \equiv \sum_{a=0}^{m} \hbar \alpha_{a} z_{a}^{k}(k=0,1, \cdots, n)$ and $c_{-k} \equiv-\sum_{a=0}^{m} \hbar \alpha_{a} z_{a}^{k}(k=1, \cdots, m)$. 
The duality transform $x_{i} \rightarrow 1 / x_{i}$ and $y_{j} \rightarrow 1 / y_{j}$ induces the dual potential

$$
\begin{aligned}
& \bar{V}_{1}(z)=\bar{b}_{0} \log z-\sum_{k>0} \frac{\bar{b}_{k}}{k z^{k}}+\sum_{k>0} \frac{\bar{b}_{-k} z^{k}}{k}, \\
& \bar{V}_{2}(z)=\bar{a}_{0} \log z-\sum_{k>0} \frac{\bar{a}_{k}}{k z^{k}}+\sum_{k>0} \frac{\bar{a}_{-k} z^{k}}{k},
\end{aligned}
$$

where $\bar{a}_{k}=-a_{-k}$ and $\bar{b}_{k}=-b_{-k}$ when $k \neq 0 . \quad \bar{c}_{0}=c_{\infty}$ and $\bar{a}_{0}=a_{\infty}$ are fixed by the neutrality condition $b_{0}+b_{\infty}+\epsilon(N-M / 2)=\epsilon$ and $a_{0}+a_{\infty}+\epsilon(M-N / 2)=\epsilon$.

The loop equation at the classical/NS limit is summarized as the following. (More details can be found in appendix A). One is the quadratic equation related with the Virasoro symmetry:

$$
X_{1}^{2}+X_{2}^{2}-X_{1} X_{2}+\epsilon\left(X_{1}^{\prime}+X_{2}^{\prime}\right)+\xi_{2}=0
$$

where $X_{1}$ and $X_{2}$ are one-point resolvents $\left(R_{1}\right.$ and $\left.R_{2}\right)$ shifted by potential whose integration variable is $x_{i}$ and $y_{i}$, respectively: $X_{1}=2\left(R_{1}+\frac{1}{3}\left(2 V_{1}^{\prime}+V_{2}^{\prime}\right)\right)$ and $X_{2}=2\left(R_{2}\right.$ $\left.+\frac{1}{3}\left(V_{1}^{\prime}+2 V_{2}^{\prime}\right)\right) \cdot \xi_{2}(z)$ is the energy momentum tensor (Virasoro current) expectation value

$$
\xi_{2}(z)=-2 \epsilon\left(V_{1}^{\prime \prime}+V_{2}^{\prime \prime}\right)-\frac{4}{3}\left(V_{1}^{\prime 2}+V_{2}^{\prime 2}+V_{1}^{\prime} V_{2}^{\prime}\right)-F=\frac{\left\langle I_{m}|T(z)| I_{n}\right\rangle}{\left\langle I_{m} \mid I_{n}\right\rangle} .
$$

where $F \equiv f_{1}+f_{2}$ is defined in the appendix and has the mode expansion $f_{1}(z)+f_{2}(z)=$ $\sum_{k=-m}^{n-1} d_{k} / z^{k+2}$. Therefore, $\xi_{2}(z)$ has the mode expansion

$$
\xi_{2}(z)=\sum_{k=-2 m}^{2 n} \frac{A_{k}}{z^{k+2}}-\sum_{k=-m}^{n-1} \frac{d_{k}}{z^{k+2}} .
$$

Here $A_{k}$ is a constant

$$
A_{k}=2 \epsilon(k+1)\left(a_{k}+b_{k}\right)-\frac{4}{3} \sum_{r+s=k}\left(a_{r} a_{s}+b_{r} b_{s}+a_{r} b_{s}\right) .
$$

Since $T(z)=\sum_{k} L_{k} / z^{k+2}, A_{k}(n \leq k \leq 2 n)$ is the eigenvalues of $L_{k}$ of the irregular state $\left|I_{n}\right\rangle$, consistent with the definition of the irregular state of rank $n$. The mode $d_{k}$ has an important role since it is related with the partition function as in the Virasoro case. When $0 \leq k \leq n-1$, one has

$$
d_{k}=v_{k}\left(F_{(m ; n)}\right), \quad v_{k}=\sum_{s>0} s\left(b_{s+k} \frac{\partial}{\partial b_{s}}+a_{s+k} \frac{\partial}{\partial a_{s}}\right) .
$$

One may find its dual form if one replaces $b_{k}\left(a_{k}\right)$ and $k \neq 0$ with $\bar{b}_{k}$ and $\bar{a}_{k}$, respectively.

The other loop equation is cubic. One may put the cubic equation conveniently into two separate equations when combined with the previous quadratic one.

$$
\begin{aligned}
& X_{1}^{3}+\xi_{2} X_{1}+3 \epsilon X_{1} X_{1}^{\prime}+\epsilon^{2} X_{1}^{\prime \prime}=+\frac{2}{3 \sqrt{3}} \xi_{3}-\frac{\epsilon}{2} \xi_{2}^{\prime}, \\
& X_{2}^{3}+\xi_{2} X_{2}+3 \epsilon X_{2} X_{2}^{\prime}+\epsilon^{2} X_{2}^{\prime \prime}=-\frac{2}{3 \sqrt{3}} \xi_{3}-\frac{\epsilon}{2} \xi_{2}^{\prime},
\end{aligned}
$$


where $\xi_{3}$ is given in terms of the coefficients of the potential:

$$
\xi_{3}(z)=\sum_{k=-3 m}^{3 n} \frac{B_{k}}{z^{k+3}}-\sum_{k=-2 m}^{2 n-1} \frac{e_{k}}{z^{k+3}} .
$$

$B_{k}$ is a constant

$$
\begin{aligned}
B_{k}= & \frac{4}{3 \sqrt{3}} \sum_{r+s+t=k}\left(2\left(b_{r} b_{s} b_{t}-a_{r} a_{s} a_{t}\right)+3\left(b_{r} b_{s} a_{t}-a_{r} a_{s} b_{t}\right)\right) \\
& -\frac{\sqrt{3}}{2} \epsilon \sum_{r+s=k}\left(2(k+2)\left(b_{r} b_{s}-a_{r} a_{s}\right)+(r-s)\left(b_{r} a_{s}-a_{r} b_{s}\right)\right) \\
& +\frac{\sqrt{3}}{2} \epsilon^{2}(k+1)(k+2)\left(b_{k}-a_{k}\right),
\end{aligned}
$$

which comes from the terms

$$
\left(\frac{4}{3 \sqrt{3}}\left(2 V_{1}^{\prime 3}+3 V_{1}^{\prime 2} V_{2}^{\prime}\right)+\sqrt{3} \epsilon\left(2 V_{1}^{\prime} V_{1}^{\prime \prime}+V_{2}^{\prime} V_{1}^{\prime \prime}\right)+\frac{\epsilon^{2}}{3} V_{1}^{\prime \prime \prime}\right)-(1 \leftrightarrow 2) .
$$

It turns out that $\xi_{3}(z)$ is the expectation of the $\mathcal{W}_{3}$ current $W(z)$

$$
\xi_{3}(z)=\frac{\left\langle I_{m}|W(z)| I_{n}\right\rangle}{\left\langle I_{m} \mid I_{n}\right\rangle}=\sum_{k} \frac{\left\langle W_{k}\right\rangle}{z^{k+3}}
$$

and $B_{k}(2 n \leq k \leq 3 n)$ is the $W_{k}$ eigenvalue of the ket (right state). When $-3 n \leq k \leq-2 n$, $B_{k}$ is the $-W_{-k}$ eigenvalue of the bra (left state) since $W_{k}^{\dagger}=-W_{-k}$ (this anti-hermiticity comes from our normalization. See appendix B.).

The moment $e_{k}$ induces the flow equation. When $n \leq k \leq 2 n-1, e_{k}$ applies to the right state

$$
e_{k}=\mu_{k}\left(F_{(m ; n)}\right), \quad \mu_{k}=\sum_{\substack{k=r+s-t ; \\ t>0}} \sqrt{3} t\left(\left(a_{r} a_{s}+2 a_{r} b_{s}\right) \frac{\partial}{\partial a_{t}}-\left(b_{r} b_{s}+2 a_{r} b_{s}\right) \frac{\partial}{\partial b_{t}}\right) .
$$

Its dual form applies to the left state.

It is worth to note that if one defines $\Psi_{i}(z)=\exp \left(\frac{1}{\epsilon} \int^{z} X_{i}\left(z^{\prime}\right) d z^{\prime}\right)$ with $i=1,2$. Then, the loop equations (3.10) and (3.11) can be rewritten as a third order differential equation of $\Psi_{i}(z)$ :

$$
\left(\epsilon^{3} \frac{\partial^{3}}{\partial z^{3}}+\xi_{2} \epsilon \frac{\partial}{\partial z}+U_{i}(z)\right) \Psi_{i}(z)=0
$$

where $U_{1}(z)=+\frac{2}{3 \sqrt{3}} \xi_{3}-\frac{\epsilon}{2} \xi_{2}^{\prime}$ and $U_{2}(z)=-\frac{2}{3 \sqrt{3}} \xi_{3}-\frac{\epsilon}{2} \xi_{2}^{\prime}$.

\subsection{Spectral curve and partition function}

As shown in section 2, the symmetry present in the spectral curve will be used to find the partition function $\mathcal{Z}_{(m ; n)}$. The loop equations (3.5), (3.10) and (3.11) are our starting point. Our first step is to introduce two monic polynomials of $z$ with degree $N$ and $M$ : $P(z)=\left\langle\prod_{i=1}^{N}\left(z-x_{i}\right)\right\rangle=\prod_{\alpha=1}^{N}\left(z-t_{\alpha}\right)$ and $Q(z)=\left\langle\prod_{j}^{M}\left(z-y_{j}\right)\right\rangle=\prod_{\alpha=1}^{M}\left(z-w_{\alpha}\right)$. At 
the classical/NS limit, one has the resolvents as rational functions: $2 R_{1}(z)=\epsilon P^{\prime}(z) / P(z)$ and $2 R_{2}(z)=\epsilon Q^{\prime}(z) / Q(z)$. We rewrite the quadratic equation (3.5) and cubic equations in (3.10) and (3.11) in terms of the polynomials $P$ and $Q$. The quadratic equation reduces to the second order differential equation:

$$
\epsilon^{2}\left(P^{\prime \prime} Q-P^{\prime} Q^{\prime}+P Q^{\prime \prime}\right)+2 \epsilon\left(V_{1}^{\prime} P^{\prime} Q+V_{2}^{\prime} P Q^{\prime}\right)=F P Q .
$$

The cubic equations reduces to the third order differential equation:

$$
\begin{aligned}
& \epsilon^{3} P^{\prime \prime \prime}+2 \epsilon^{2}\left(2 V_{1}^{\prime}+V_{2}^{\prime}\right) P^{\prime \prime}+\epsilon\left(4 V_{1}^{\prime}\left(V_{1}^{\prime}+V_{2}^{\prime}\right)+2 \epsilon V_{1}^{\prime \prime}-F\right) P^{\prime}=G_{1} P \\
& \epsilon^{3} Q^{\prime \prime \prime}+2 \epsilon^{2}\left(V_{1}^{\prime}+2 V_{2}^{\prime}\right) Q^{\prime \prime}+\epsilon\left(4 V_{2}^{\prime}\left(V_{1}^{\prime}+V_{2}^{\prime}\right)+2 \epsilon V_{2}^{\prime \prime}-F\right) Q^{\prime}=G_{2} Q .
\end{aligned}
$$

where

$$
\begin{aligned}
& G_{1}=\sum_{k=-2 m}^{2 n-1} \frac{1}{z^{k+3}}\left\{-\frac{2}{3 \sqrt{3}} e_{k}+\frac{2}{3} \sum_{r+s=k} d_{r}\left(2 b_{s}+a_{s}\right)\right\}-\frac{\epsilon}{2} \sum_{k=-m}^{n-1} \frac{(k+2) d_{k}}{z^{k+3}}, \\
& G_{2}=\sum_{k=-2 m}^{2 n-1} \frac{1}{z^{k+3}}\left\{+\frac{2}{3 \sqrt{3}} e_{k}+\frac{2}{3} \sum_{r+s=k} d_{r}\left(2 a_{s}+b_{s}\right)\right\}-\frac{\epsilon}{2} \sum_{k=-m}^{n-1} \frac{(k+2) d_{k}}{z^{k+3}} .
\end{aligned}
$$

Our next step is to find the mode $d_{a}(0 \leq a \leq n-1)$ and $e_{k}(n \leq k \leq 2 n-1)$ and their duals if necessary. As noted in section 2 , it is not easy to find the exact form of $d_{a}$ and and $e_{k}$. We provide some examples of the partition function using the $\epsilon$ expansion method. Note that $R_{1}, R_{2}$ and $F$ are $O(\epsilon)$ whereas $V_{1}$ and $V_{2}$ are $O(1)$. Therefore, denoting $R_{i}=\sum_{k \geq 1} R_{i}^{(k)} \epsilon^{k}, F=\sum_{k \geq 1} F^{(k)} \epsilon^{k}$ and $G_{i}=\sum_{k \geq 1} G_{i}^{(k)} \epsilon^{k}$, we have $R_{1}^{(1)}$ and $R_{2}^{(1)}$ at the leading order of the loop equations (3.19) and (3.20):

$$
2 R_{1}^{(1)}=\frac{G_{1}^{(1)}}{4 V_{1}^{\prime}\left(V_{1}^{\prime}+V_{2}^{\prime}\right)}, \quad 2 R_{2}^{(1)}=\frac{G_{2}^{(1)}}{4 V_{2}^{\prime}\left(V_{1}^{\prime}+V_{2}^{\prime}\right)} .
$$

The stationary point of the potentials, $V_{1}^{\prime}=0, V_{2}^{\prime}=0$ and $V_{1}^{\prime}+V_{2}^{\prime}=0$ provide the pole structure of the resolvents (zeros of the polynomials $P$ and $Q$ ). This is the reminiscence of the cut structure on the Riemann sheet which appears at large $N$ limit. Let us denote the number of poles of the resolvent $R_{1}$ by $N_{k}$ and $R_{2}$ by $M_{k}$ so that $N=\sum_{k=1}^{2 n} N_{k}$ and $M=\sum_{k=1}^{2 n} M_{k}$. There are equal number of stationary points for $V_{1}$ and $V_{2}$. Therefore, we have identities from the filling fractions. When $1 \leq k \leq n$, we have

$$
\oint_{\mathcal{A}_{k}} d z \frac{G_{1}^{(1)}}{4 V_{1}^{\prime}\left(V_{1}^{\prime}+V_{2}^{\prime}\right)}=N_{k}, \quad \oint_{\mathcal{B}_{k}} d z \frac{G_{2}^{(1)}}{4 V_{2}^{\prime}\left(V_{1}^{\prime}+V_{2}^{\prime}\right)}=M_{k},
$$

where the contours $\mathcal{A}_{k}$ and $\mathcal{B}_{k}$ encircle the stationary points of $V_{1}$ and $V_{2}$, respectively. When $n+1 \leq k \leq 2 n$, we have

$$
\oint_{\mathcal{C}_{k}} d z \frac{G_{1}^{(1)}}{4 V_{1}^{\prime}\left(V_{1}^{\prime}+V_{2}^{\prime}\right)}=N_{k}, \quad \oint_{\mathcal{C}_{k}} d z \frac{G_{2}^{(1)}}{4 V_{2}^{\prime}\left(V_{1}^{\prime}+V_{2}^{\prime}\right)}=M_{k},
$$

where $\mathcal{C}_{k}$ encircles the stationary point of $V_{1}+V_{2}$. It turns out that $N_{k}=M_{k}$ since $R_{1}-R_{2}$ has no poles inside $\mathcal{C}_{k}$. These identities provide $3 n$-independent equations which solve $d_{a}$ 
and $e_{k}$ in terms of the filling fraction at the lowest order in $\epsilon$. One obtains the non-trivial contribution from the next order

$$
\begin{aligned}
2 R_{1}^{(2)}= & \frac{G_{1}^{(2)}}{4 V_{1}^{\prime}\left(V_{1}^{\prime}+V_{2}^{\prime}\right)}+\frac{\left(F^{(1)}-2 V_{1}^{\prime \prime}\right) G_{1}^{(1)}-2\left(2 V_{1}^{\prime}+V_{2}^{\prime}\right)\left(G_{1}^{(1)}\right)^{\prime}}{16\left(V_{1}^{\prime}\left(V_{1}^{\prime}+V_{2}^{\prime}\right)\right)^{2}} \\
& +\frac{\left(2 V_{1}^{\prime}+V_{2}^{\prime}\right) V_{1}^{\prime \prime} G_{1}^{(1)}}{8\left(V_{1}^{\prime}\right)^{3}\left(V_{1}^{\prime}+V_{2}^{\prime}\right)^{2}}+\frac{\left(2 V_{1}^{\prime}+V_{2}^{\prime}\right)\left(V_{1}^{\prime \prime}+V_{2}^{\prime \prime}\right) G_{1}^{(1)}}{8\left(V_{1}^{\prime}\right)^{2}\left(V_{1}^{\prime}+V_{2}^{\prime}\right)^{3}}-\frac{\left(2 V_{1}^{\prime}+V_{2}^{\prime}\right)\left(G_{1}^{(1)}\right)^{2}}{32\left(V_{1}^{\prime}\left(V_{1}^{\prime}+V_{2}^{\prime}\right)\right)^{3}} .
\end{aligned}
$$

$R_{2}^{(2)}$ is obtained if $V_{1}$ and $V_{2}$ are exchanged and $G_{1}^{(i)} \rightarrow G_{2}^{(i)}$.

\subsection{Partition function $\mathcal{Z}_{(0 ; n)}$}

The irregular partition function $\mathcal{Z}_{(0 ; n)}$ has the potential with logarithmic and inverse powers only:

$$
V_{1}(z)=b_{0} \log z-\sum_{k=1}^{n} \frac{b_{k}}{k z^{k}}, \quad V_{2}(z)=a_{0} \log z-\sum_{k=1}^{n} \frac{a_{k}}{k z^{k}} .
$$

This partition function is the two-point correlation of one regular vertex at infinity and one irregular vertex at origin and is therefore, considered as the inner product between a regular state and an irregular state.

The partition function is the function of $2 n$-variables, $\left\{b_{1}, \cdots, b_{n}\right\}$ and $\left\{a_{1}, \cdots, a_{n}\right\}$ and $d_{a}(0 \leq a \leq n-1)$ and $e_{a}(n \leq a \leq 2 n-1)$ provide $2 n$-flow equations. In this case, $d_{0}$ is simple to find: $d_{0}=2 \epsilon\left(b_{0} N+a_{0} M\right)+\epsilon^{2}(N(N-1)+M(M-1)-N M)$ if one uses the large $z$ expansion of the quadratic loop equation. Other quantities need more elaborate evaluation.

Let us consider the partition function $\mathcal{Z}_{(0 ; 1)}$, the rank 1 case. We need $e_{1}$. Using the results (3.24), (3.24) and (3.26), one finds $e_{1}$ :

$$
\begin{aligned}
e_{1}=2 \sqrt{3} \epsilon & {\left[\left(a_{1}+2 b_{1}\right)\left(a_{0} M+b_{0} N\right)+3 b_{0}\left(a_{0}+b_{0}\right)\left(N-N_{1}\right) \xi_{1}+3 b_{0}\left(a_{0}+b_{0}\right) N_{1} \xi_{3}\right] } \\
+\sqrt{3} \epsilon^{2}\left[-\frac{3 a_{0} \xi_{3}\left(\left(N^{2}+2 N_{1}\left(N_{1}+M+1\right)-N\left(4 N_{1}+M+1\right)\right) \xi_{1}+N_{1}\left(N_{1}-1\right) \xi_{3}\right)}{\xi_{1}-\xi_{3}}\right. & \\
& \left.+3 b_{0}\left(\left(N^{2}+N_{1}\left(N_{1}+3\right)-N\left(2 N_{1}+3\right)\right) \xi_{1}+N_{1}\left(N_{1}-1\right) \xi_{3}\right)+\left(a_{1}+2 b_{1}\right) d_{0}^{(2)}\right]+\mathcal{O}\left(\epsilon^{3}\right),
\end{aligned}
$$

where $d_{0}^{(2)}=N(N-1)+M(M-1)-N M$ is the $\epsilon^{2}$-order coefficient of $d_{0}$. In addition, $\xi_{1}=-\frac{b_{1}}{b_{0}}$ and $\xi_{3}=-\frac{a_{1}+b_{1}}{a_{0}+b_{0}}$ are stationary points of $V_{1}$ and $V_{1}+V_{2}$, respectively. Using the flow equations $d_{0}=v_{0}\left(F_{(0: 1)}\right)$ and $e_{1}=\mu_{1}\left(F_{(0 ; 1)}\right)$, one can find the free energy and the partition function up to $O\left(\epsilon^{2}\right)$

$$
\begin{aligned}
\mathcal{Z}_{(0 ; 1)=} & a_{1}^{-\left(2 \epsilon a_{0} M_{1}+\epsilon^{2} M_{1}\left(M_{1}-1\right)\right) / \hbar^{2}} b_{1}^{-\left(2 \epsilon b_{0} N_{1}+\epsilon^{2} a_{0} N_{1}\left(N_{1}-1\right)\right) / \hbar^{2}}\left(a_{1}+b_{1}\right)^{-\left(2 \epsilon N_{2}\left(a_{0}+b_{0}\right)+\epsilon^{2} N_{2}\left(N_{2}-3\right)\right) / \hbar^{2}} \\
& \times\left(a_{0} b_{1}-a_{1} b_{0}\right)^{-\epsilon^{2}\left(N_{1} N_{2}+N_{2}+M_{1} N_{2}-M_{1} N_{1}\right) / \hbar^{2}} .
\end{aligned}
$$




\subsection{Partition function $\mathcal{Z}_{(m ; n)}$ and ICB}

As in the Virasoro case, $\mathcal{Z}_{(m ; n)}$ is equivalent to the two-point correlation of irregular vertex operators. One can evaluate the irregular conformal block (ICB) using the relation with the partition function [36]

$$
\mathcal{F}_{\Delta}^{(m ; n)}\left(\left\{a_{-k}, b_{-k}: a_{k}, b_{k}\right\}\right)=\frac{e^{\zeta_{(m ; n)} \mathcal{Z}_{(m ; n)}\left(a_{0}, b_{0} ;\left\{a_{\ell}, b_{\ell}\right\}\right)}}{\left.\mathcal{Z}_{(0 ; n)}\left(a_{0}, b_{0} ;\left\{a_{k}, b_{k}\right\}\right) \mathcal{Z}_{(0 ; m)}\left(\bar{a}_{0}, \bar{b}_{0} ;\left\{\bar{a}_{k}, \bar{b}_{k}\right\}\right)\right)} .
$$

This time the extra factor $e^{\zeta_{(m ; n)}}$ is the generalization of the Virasoro case: $\hbar^{2} \zeta_{(m: n)}=$ $-\sum_{k}^{\min (m, n)} \frac{4}{3 k}\left(2 a_{k} a_{-k}+a_{k} b_{-k}+b_{k} a_{-k}+2 b_{k} b_{-k}\right)$.

We find the partition function $\mathcal{Z}_{(1 ; 1)}$ using the $\epsilon$ expansion. By rescaling $x_{i} \rightarrow x_{i} / a_{-1}$ and $y_{j} \rightarrow y_{j} / a_{-1}$, one obtains $a_{-1}$ dependence and has the partition function with three parameters, $\eta_{0} \equiv a_{1} a_{-1}, t_{1} \equiv b_{1} / a_{1}$ and $t_{-1} \equiv b_{-1} / a_{-1}$. Then, the partition function is to be evaluated from three flow equations: $d_{0}=v_{0}\left(F_{(1 ; 1)}\right), e_{1}=\mu_{1}\left(F_{(1 ; 1)}\right)$ and $e_{-1}=$ $\mu_{-1}\left(F_{(1 ; 1)}\right)+\nu_{-1}^{c}$ where $\nu_{-1}^{c}$ is a constant (see appendix A). We use notations for the filling fractions as $M_{0}=M_{1}+M_{2}, N_{0}=N_{1}+N_{2}\left(M_{2}=N_{2}\right)$ and $M_{\infty}=M_{-1}+M_{-2}$, $N_{\infty}=N_{-1}+N_{-2}\left(M_{-2}=N_{-2}\right)$ with $M=M_{0}+M_{\infty}, N=N_{0}+N_{\infty}$. We have up to $\mathcal{O}\left(\epsilon^{1}\right)$,

$$
\begin{aligned}
d_{0}=2 \epsilon\left(a_{0} M_{0}+b_{0} N_{0}\right)+\mathcal{O}\left(\eta_{0}\right) & \\
e_{1}=2 \sqrt{3} \epsilon[ & a_{0}\left(M_{0} a_{1}+\left(2 M_{0}-3 N_{0}+3 N_{1}\right) b_{1}\right) \\
+ & \left.b_{0}\left(\left(N_{0}-3 N_{1}\right) a_{1}-N_{0} b_{1}\right)\right]+\mathcal{O}\left(\eta_{0}\right), \\
e_{-1}-\nu_{-1}^{c}=-2 \sqrt{3} \epsilon & {\left[a_{0}\left(M_{\infty} a_{-1}+\left(2 M_{\infty}-3 N_{\infty}+3 N_{-1}\right) b_{-1}\right)\right.} \\
& \left.+b_{0}\left(\left(N_{\infty}-3 N_{-1}\right) a_{-1}-N_{\infty} b_{-1}\right)\right]+\mathcal{O}\left(\eta_{0}\right),
\end{aligned}
$$

and the partition function

$$
\begin{aligned}
& \mathcal{Z}_{(1 ; 1)}= a_{1}^{-\left(2 \epsilon a_{0} M_{1}\right) / \hbar^{2}} a_{-1}^{-\left(2 \epsilon a_{\infty} M_{-1}\right) / \hbar^{2}} b_{1}^{-\left(2 \epsilon b_{0} N_{1}\right) / \hbar^{2}} b_{-1}^{-\left(2 \epsilon b_{\infty} N_{-1}\right) / \hbar^{2}} \\
& \times\left(a_{1}+b_{1}\right)^{-2 \epsilon\left(a_{0}+b_{0}\right) / \hbar^{2}}\left(a_{-1}+b_{-1}\right)^{-2 \epsilon\left(a_{\infty}+b_{\infty}\right) / \hbar^{2}} \\
& \times \exp \left[\frac { 2 \epsilon \eta _ { 0 } } { \hbar ^ { 2 } a _ { 0 } b _ { 0 } ( a _ { 0 } + b _ { 0 } ) } \left\{b_{0}^{2}\left(M_{1}-M_{-1}\right)+a_{0}^{2}\left(N_{1}-N_{-1}\right) t_{1} t_{-1}+a_{0} b_{0}\left(M_{1}-M_{-1}\right.\right.\right. \\
&\left.\left.\left.\quad+\left(N_{2}-N_{-2}\right)\left(1+t_{1}+t_{-1}\right)+\left(N_{1}+N_{2}-N_{-1}-N_{-2}\right) t_{1} t_{-1}\right)\right\}+\mathcal{O}\left(\eta_{0}^{2}\right)\right] .
\end{aligned}
$$

This provides ICB

$$
\begin{aligned}
\mathcal{F}_{\Delta}^{(1 ; 1)}= & {\left[1-\frac{4 \eta_{0}}{3 \hbar^{2}}\left(2+t_{1}+t_{-1}+2 t_{1} t_{-1}\right)+\mathcal{O}\left(\eta_{0}^{2}\right)\right]+\epsilon\left[\frac { 2 \eta _ { 0 } } { \hbar ^ { 2 } a _ { 0 } b _ { 0 } ( a _ { 0 } + b _ { 0 } ) } \left\{b_{0}^{2}\left(M_{1}-M_{-1}\right)\right.\right.} \\
& +a_{0}^{2}\left(N_{1}-N_{-1}\right) t_{1} t_{-1}+a_{0} b_{0}\left(M_{1}-M_{-1}+\left(N_{2}-N_{-2}\right)\left(1+t_{1}+t_{-1}\right)\right. \\
& \left.\left.\left.+\left(N_{1}+N_{2}-N_{-1}-N_{-2}\right) t_{1} t_{-1}\right)\right\}+\mathcal{O}\left(\eta_{0}^{2}\right)\right]+\mathcal{O}\left(\epsilon^{2}\right) .
\end{aligned}
$$


One may use the perturbative method to find ICB using IMM with the relation (3.30). One may put the reference potentials $V^{(0)}$ and its perturbations $\Delta V^{(0)}$ for $N_{0}$ and $M_{0}$ variables:

$$
\begin{aligned}
V^{(0)}\left(x_{I}, y_{J}\right) & =\sum_{I=1}^{N_{0}}\left(b_{0} \log x_{I}-\sum_{k=1}^{n} \frac{b_{k}}{k x_{I}^{k}}\right)+\sum_{J=1}^{M_{0}}\left(a_{0} \log y_{J}-\sum_{k=1}^{m} \frac{a_{k}}{k y_{J}^{k}}\right), \\
\Delta V^{(0)}\left(x_{I}, y_{J}\right) & =\sum_{I=1}^{N_{0}}\left(\sum_{\ell=1}^{n} \frac{b_{-\ell}}{\ell} x_{I}^{\ell}\right)+\sum_{J=1}^{M_{0}}\left(\sum_{\ell=1}^{n} \frac{a_{-\ell}}{\ell} y_{J}^{\ell}\right) .
\end{aligned}
$$

For the rest variables, $N_{\infty}$ and $M_{\infty}$ variables, one has the reference potential $V^{(\infty)}\left(\mu_{K}, \nu_{L}\right)$ and perturbation $\Delta V^{(\infty)}\left(\mu_{K}, \nu_{L}\right)$ which can be put into the similar form $V^{(0)}, \Delta V^{(0)}$ with dual variables if one uses the dual transformation $\mu_{K} \rightarrow 1 / \mu_{K}$ and $\nu_{L} \rightarrow 1 / \nu_{L}$. After this, one has ICB in the following form

$$
\begin{aligned}
\mathcal{F}_{\Delta}^{(m ; n)}\left(\left\{a_{-k}, b_{-k}: a_{k}, b_{k}\right\}\right)=e^{\zeta_{(m ; n)}} & \left\langle\prod_{I, K}\left(1-x_{I} \mu_{K}\right)^{2 \beta} \prod_{J, L}\left(1-y_{J} \nu_{L}\right)^{2 \beta} \prod_{I, L}\left(1-x_{I} \nu_{L}\right)^{-\beta}\right. \\
& \left.\times \prod_{J, K}\left(1-y_{J} \mu_{K}\right)^{2 \beta} e^{\frac{\sqrt{\beta}}{g}\left(\Delta V^{(0)}\left(x_{I}, y_{J}\right)+\Delta V^{(\infty)}\left(\mu_{K}, \nu_{L}\right)\right)}\right\rangle_{0},
\end{aligned}
$$

where the bracket denotes the expectation value with respect to the reference potentials, $V^{(0)}$ and $V^{(\infty)}$. One may obtain the expectation values using the large $z$ expansion of the resolvents in the loop equations (3.10) and (3.11) of the reference partition functions.

We find $\mathcal{F}_{\Delta}^{(1 ; 1)}$ as the simplest example. Up to the first order of $a_{1}$ and $b_{1}$ (also their duals $\bar{a}_{1}$ and $\bar{b}_{1}$ ), we have

$$
\begin{aligned}
\mathcal{F}_{\Delta}^{(1 ; 1)}=1+\frac{1}{\hbar^{2}}[ & 2 \epsilon^{2}\left(\left\langle x_{I}\right\rangle\left\langle\mu_{K}\right\rangle+\left\langle y_{J}\right\rangle\left\langle\nu_{L}\right\rangle\right)-\epsilon^{2}\left(\left\langle x_{I}\right\rangle\left\langle\nu_{L}\right\rangle+\left\langle y_{J}\right\rangle\left\langle\mu_{K}\right\rangle\right) \\
& \left.+2 \epsilon\left(\bar{b}_{1}\left\langle x_{I}\right\rangle+\bar{a}_{1}\left\langle y_{J}\right\rangle+b_{1}\left\langle\mu_{K}\right\rangle+a_{1}\left\langle\nu_{L}\right\rangle\right)+\frac{4}{3}\left(2 a_{1} \bar{a}_{1}+a_{1} \bar{b}_{1}+b_{1} \bar{a}_{1}+2 b_{1} \bar{b}_{1}\right)\right] .
\end{aligned}
$$

Here we omitted summation symbols inside the expectation value bracket for simplicity. Each expectation values can be read off from the order of $z^{-4}$ of the loop equations (3.10) and (3.11) for the reference partition function $\mathcal{Z}_{(0 ; 1)}$. Finally, we obtain ICB at the first order of $a_{1}$ and $b_{1}$

$$
\begin{aligned}
\mathcal{F}_{\Delta}^{(1 ; 1)}= & 1-\frac{1}{9 \hbar^{2}\left(4 \omega_{0}^{2}+\Delta^{2}\left(4 \Delta-3 \epsilon^{2}\right)\right)}\left[8 \Delta \omega_{-1} \omega_{1}\right. \\
& \left.+12 \omega_{0}\left(\omega_{-1} \ell_{1}+\omega_{1} \ell_{-1}\right)-\frac{9}{2} \Delta \ell_{-1} \ell_{1}\left(4 \Delta-3 \epsilon^{2}\right)\right]
\end{aligned}
$$

where $\Delta=-\frac{4}{3}\left(\alpha^{2}+\alpha \beta+\beta^{2}\right)+2 \epsilon(\alpha+\beta)$ with $\alpha=a_{0}+\epsilon\left(M_{0}-N_{0} / 2\right), \beta=b_{0}+\epsilon\left(N_{0}-M_{0} / 2\right)$, $\ell_{1}=-\frac{4}{3}\left(a_{0}\left(2 a_{1}+b_{1}\right)+b_{0}\left(a_{1}+2 b_{1}\right)\right)+4 \epsilon\left(a_{1}+b_{1}\right)$ and its dual $\ell_{-1}$ are the constant modes of $\xi_{2}(z)$. As in the Virasoro case, we may identify the expectation values of the Virasoro 
generators: $\ell_{1}=\frac{\left\langle\Delta\left|L_{1}\right| I^{(1)}\right\rangle}{\left\langle\Delta \mid I^{(1)}\right\rangle}, \ell_{-1}=\frac{\left\langle I^{(1)}\left|L_{-1}\right| \Delta\right\rangle}{\left\langle I^{(1)} \mid \Delta\right\rangle}$ and $\Delta=\frac{\left\langle\Delta\left|L_{0}\right| I^{(1)}\right\rangle}{\left\langle\Delta \mid I^{(1)}\right\rangle}$ where $\left\langle\Delta \mid I^{(1)}\right\rangle$ is $\mathcal{Z}_{(0 ; 1)}$ and $\left\langle I^{(1)} \mid \Delta\right\rangle$ is $\overline{\mathcal{Z}}_{(0 ; 1)}$. In addition, $\omega_{k}$ is the mode appearing in $\xi_{3}(z)$. The constant mode $\omega_{0}=\frac{1}{3 \sqrt{3}}(\alpha-\beta)(4 \alpha+2 \beta-3 \epsilon)(2 \alpha+4 \beta-3 \epsilon)$ is identified as $\frac{\left\langle\Delta\left|W_{0}\right| I^{(1)}\right\rangle}{\left\langle\Delta \mid I^{(1)}\right\rangle}$ and the other modes are expectation values: $\omega_{1}=\frac{\left\langle\Delta\left|W_{1}\right| I^{(1)}\right\rangle}{\left\langle\Delta \mid I^{(1)}\right\rangle}=B_{1}-e_{1}$ and $\omega_{-1}$ is its dual. We check that the $\epsilon$ expansion of the above $\mathcal{F}_{\Delta}^{(1 ; 1)}$ in (3.39) is in complete agreement with (3.35). It is also noted that ICB is manifestly dual invariant.

ICB of (3.39), obtained from the perturbation of the irregular matrix model is convenient to find the irregular state in terms of descendants. The irregular state of the rank 1 has the form

$$
\begin{aligned}
\frac{\left|I^{(1)}\right\rangle}{\left\langle\Delta \mid I^{(1)}\right\rangle}= & 1-\frac{1}{9 \hbar\left(\omega_{0}^{2}+\Delta^{2}\left(\Delta-\frac{c-2}{32}\right)\right)}\left[\left(2 \Delta \omega_{1}-3 \omega_{0} \ell_{1}\right) W_{-1}|\Delta\rangle\right. \\
& \left.-\left(3 \omega_{0} \omega_{1}+\frac{9}{2} \Delta\left(\Delta-\frac{c-2}{32}\right) \ell_{1}\right) L_{-1}|\Delta\rangle\right]+\cdots
\end{aligned}
$$

where $c=2+24 \epsilon^{2}$ is the central charge and $\cdots$ refers to the higher descendant. The irregular state (3.40) has no semi-degenerate condition at the first level in contrast to the state constructed in $[30,41,42]$ where $L_{-1}$ is related to $W_{-1}$ descendant. Instead, the coefficient $\omega_{1}$ is not a simple constant and is given in terms of the flow equation with respect to the proper normalization $\mathcal{Z}_{(0: 1)}$. This feature also appeared in Virasoro irregular state with rank 2 and higher [20]. However, here in Toda irregular state, the non-trivial feature appears even for the rank 1 and at the first descendant level.

\section{Relation with gauge theories}

The Nekrasov partition function for $\mathcal{N}=2$ is given as the product of the perturbative part and instanton part:

$$
Z_{\text {full }}(\vec{q} ; \vec{a}, m ; \epsilon)=Z_{\text {perturb }} Z_{\text {inst }}=Z_{\text {tree }} Z_{1 \text { loop }} Z_{\text {inst }} .
$$

According to AGT, the Nekrasov partition function of $\mathrm{U}(N)$ gauge theory with $N_{f}=2 N$ is related with the correlation function of Toda field theory. Explicitly, the correlation function on a sphere with $n+3$ punctures corresponds to a linear quiver $n$ of $\mathrm{SU}(N)$ gauge groups:

$$
\left\langle V_{n}(\infty) V_{n-1}(1) V_{n-2}\left(q_{1}\right) \cdots V_{2}\left(q_{1} \cdots q_{n-3}\right) V_{1}(0)\right\rangle \propto \int\left|Z_{\text {full }}(\vec{q} ; \vec{a}, m ; \epsilon)\right|^{2}
$$

Especially the instanton part of Nekrasov partition function $Z_{\text {inst }}$ corresponds to the conformal blocks $\mathcal{F}_{\Delta}$ in the CFT side; $Z_{\text {inst }}=\mathcal{F}_{\Delta}$. One may try to prove this conjecture by evaluating the conformal block directly [19, 31, 32, 43].

One may equally check the AGT conjecture by comparing the Seiberg-Witten curve of the gauge theory with the corresponding spectral curve from CFT side. Seiberg-Witten 
curve of $\mathrm{SU}(N)$ gauge theory with $N_{f}=2 N$ is given in [44-46],

$$
x^{n}=\sum_{k=2}^{n} \frac{P_{2 k}^{(k)}(z)}{(z(z-1)(z-q)))^{k}} x^{n-k},
$$

where $P_{2 k}(z)$ is a $2 k$ polynomial in $z$ and $q=e^{i \pi \tau}$ with $\tau$ the gauge coupling constant $\tau=\theta / \pi+8 \pi i / g^{2}$.

On the other hand, from the matrix model with $A_{n-1}$ quiver one may have the spectral curve of the type

$$
x^{n}=\sum_{k=2}^{n}(-1)^{k-1} \phi_{k}(z) x^{n-k},
$$

where $\phi_{k}(z)$ is the expectation value of the conformal current with dimension $k$. This was obtained from the the expectation value of the Miura transform of $\operatorname{det}(x-i \hbar \partial \varphi(z))=0$ with Toda field $\varphi$ in the matrix model context. Even though this spectral curve holds at $\epsilon \rightarrow 0$ limit (Note that this limit corresponds to the large $N$ limit where $N$ is the number of the eigenvalues of the matrix), one may identify parameters between two theories so that the pole structure of the curves matches each other as done in [44]. One may have more accurate spectral curve if one uses the loop equation of the matrix model.

This conjecture still works for irregular conformal blocks. Gaiotto [15] obtained the irregular singularity for the simplest example of $\mathcal{W}_{2}$ and identify the result with the asymptotically free theories of $\mathrm{SU}(2)$ gauge theory with $N_{f}<4$. Explicitly, $\mathrm{SU}(2)$ gauge theory with $N_{f}=4$ reduces to $\mathrm{SU}(2)$ pure Yang-Mills theory if the masses $\mu_{i}$ of hyper-multiplets are to infinity $\mu_{1}, \ldots, \mu_{4} \rightarrow \infty$ and $q \rightarrow 0$, while keeping $q \prod_{I=1}^{4} \mu_{I}=\Lambda^{4}$. Then the Seiberg-Witten curve is given as $x^{2}=\phi_{2}(z)$ where

$$
\phi_{2}=\frac{\Lambda^{2}}{z^{3}}+\frac{2 u}{z^{2}}+\frac{\Lambda^{2}}{z},
$$

which contains the strongest irregular singularity with odd power on the Riemann sphere. $u$ parameterizes the Coulomb branch. This irregular singularity of odd power is not properly represented in terms of matrix model as yet unless one considers the special limit from the even power singularity case so that the highest even power term vanishes.

For $N_{f}=2$, one may have the SW curve with the irregular singularity with degree 4

$$
\phi_{2}=\frac{\Lambda^{2}}{z^{4}}+\frac{2 m \Lambda}{z^{3}}+\frac{2 u}{z^{2}}+\frac{2 \tilde{m} \Lambda}{z}+\Lambda^{2}
$$

where $q \mu_{2} \mu_{4}=4 \Lambda^{2}, m=\mu_{1}-\frac{\epsilon}{2}$ and $\tilde{m}=\mu_{3}-\frac{\epsilon}{2}$. In this case $\phi_{2}$ is $\xi_{2}$ in (2.5) with $n=m=1$, and the Nekrasov instanton partition function is checked to be the same as the partition function $Z_{(1 ; 1)}$ of the irregular matrix model of the rank $(1 ; 1)$ [47, 48]. In addition, $Z_{(1 ; 1)}$ corresponds to the inner product of two irregular states whose eigenvalues of $L_{2}$ and $L_{1}$ are given from $\phi_{2} ; \Lambda^{2}$ and $2 m \Lambda$ for the ket (irregular module) and $\Lambda^{2}$ and $2 \tilde{m} \Lambda$ for the bra, respectively. The Coulomb branch parameter $u$ is related to $d_{0}$ and is fixed by the filling fraction of the matrix model, the contour integral of the resolvent, which is identified with the contour integral of the Seiberg-Witten one-form $x d z$. In addition, from the fixed 
$d_{0}$ the partition function is obtained from the corresponding Virasoro flow equation as seen in section 2. Considering this result, one may naturally assume that the AGT conjecture works for the colliding limit as well with higher ranks $(m ; n)$. For the special case $(0 ; n)$, one has the irregular singularity of type $D_{2 n}$ considered in $[12,37]$ which is associated with a regular puncture of degree 2 and an irregular puncture of degree $2 n+2$ on the sphere. Specifically, $Z_{(0 ; 1)}$ corresponds to $D_{2}$ and $Z_{(0 ; 2)}$ corresponds to $D_{4}$.

One may extend the analysis to the case with $\mathcal{W}_{N}$ symmetry. For $\mathcal{W}_{3}$, one has the SW curve with the cubic equation. One can have the same spectral curve from the Penner-type matrix model with the multi-log potential

$$
V_{1}(z)=\sum_{k=1}^{p} m_{k} \log \left(q_{k}-z\right), \quad V_{2}(z)=\sum_{k=1}^{p} \tilde{m}_{k} \log \left(q_{k}-z\right),
$$

which corresponds to the linear quiver $p-2 \mathrm{SU}(3)$ gauge theories [46]. For instance, in [44], the potential (4.7) for $p=3$ was considered, which corresponds to SU(3) gauge theory with six massive flavors. The relation between six mass parameters and parameters in the potential was made by using the residue relation of the one-form $x d z$.

At the colliding limit, we used the potential (3.2) and obtained the two cubic equations (3.10) and (3.11). In fact, the two cubic equations reduce to one in the large $N$ limit $(\epsilon \rightarrow 0)$,

$$
x^{3}+\frac{\xi_{2}}{4} x-\frac{\xi_{3}}{12 \sqrt{3}}=0,
$$

where $x=2 X_{1}$ or $-2 X_{2}$. Note that the spectral curve (4.8) has the same form even before the colliding limit, where the explicit form of $\xi_{2}, \xi_{3}$ is different. At the colliding limit, $\xi_{2}$ and $\xi_{3}$ have irregular singularities which indicate positive modes of the Virasoro and $\mathcal{W}_{3}$ currents as we have shown in (3.6) and (3.15). In addition, the spectral curve provides further information about the gauge theory with the same SW curve. For example, if one considers the potential (3.27) of the rank $(0 ; n)$, then the spectral curve (at $\epsilon \rightarrow 0$ limit) is identified with the SW curve of the type IV Argyres-Douglas theory [49]:

$$
\begin{aligned}
x^{3} & +\left(\frac{v_{1}}{z^{2 n+2}}+\frac{v_{2}}{z^{2 n+1}}+\cdots+\frac{v_{2 n}}{z^{3}}+\frac{v_{2 n+1}}{z^{2}}\right) x \\
& +\left(\frac{1}{z^{3 n+3}}+\frac{\omega}{z^{3 n+2}}+\frac{u_{1}}{z^{3 n+1}}+\cdots+\frac{u_{3 n-2}}{z^{4}}+\frac{u_{3 n-1}}{z^{3}}\right)=0,
\end{aligned}
$$

where the dominant singular part in $\xi_{3}$ is normalized as 1. Comparing this SW curve with the explicit expression $\xi_{2}$ in (3.7) and $\xi_{3}$ in (3.12), one notes that $(2 n+1)$ parameters $\left(v_{1}, \cdots, v_{n+1}\right.$ and $\left.\omega, u_{1}, \cdots, u_{n-1}\right)$ are fixed by the $(2 n+2)$ parameters of the potential (3.27) with the proper normalization. The mass parameters $v_{2 n+1}$ and $u_{3 n-1}$ are determined by the additional constants $d_{0}$ and $e_{0}$.

Finally, the Coulomb branch parameters $v_{n+2}, \cdots, v_{2 n}$ and $u_{n}, \cdots, u_{3 n-2}$ are related with the contour integral of the SW one-form in the gauge theory side. From the matrix model side, the Coulomb branch parameters are given in terms of $d_{i}(i=1, \cdots, n-1)$ and $e_{j}(j=1, \cdots, 2 n-1)$ and can be fixed by $(3 n-2)$ independent filling fractions which is related with the cut structure of the spectral curve. Once the Coulomb branch parameter 
are known, one can find the partition function since the values $d_{k}$ and $e_{k}$ are directly related with the flow equations, which are the strong merit of the matrix model approach.

\section{Conclusion and outlook}

We develop a new mechanism to evaluate the irregular conformal block using the Virasoro and $\mathrm{W}$ symmetry. We use the loop equation of the irregular matrix model which encodes all the details of the conformal symmetry. At the classical/NS limit, the loop equation does not contain the multi-point resolvent terms and reduces to the simple spectral curve which contains the first derivative of the resolvent. The special feature of the spectral curve is that it contains not only constants of motion but also flow equations corresponding to the conformal symmetry. The flow equations are defined on the parameter space of the potential of the irregular matrix model, and its generators represent the Virasoro and W symmetry. We present the details of the flow equations and how to obtain the partition function and irregular conformal block. The irregular conformal block is related with the partition function of the Argyres-Douglas theory according to AGT conjecture, if one uses the parameter relations between these two theories whose details can be found, for example, in [30].

It is noted that the spectral curve and flow equation are not restricted to the irregular conformal block. The method can be applied to the regular conformal block at the classi$\mathrm{cal} / \mathrm{NS}$ limit. Using the similar flow equation, one can find the partition function $[22,40]$. Even though the partition function is simply obtained, the relation of the positions of the primary operators is not. For example, 5-point Liouville conformal block with one degenerate operator reduces to Painlevé VI as presented in [50]. It seems to be worthwhile to investigate the connection between the positions of the multi-point regular conformal block.

Nekrasov partition function and its counter part, regular conformal block are represented in terms of Young diagrams [3, 4, 43]. Irregular conformal block should also be represented in the same way, which is not well understood yet. On the other hand, conformal symmetry is reinstated in the degenerate double affine Hecke algebra(DDAHA) and Nekrasov partition function was studied in terms of DDAHA [51]. In the same way, the irregular conformal block can be better understood using DDAHA. There was a few attempts to investigate this connection $[33,34]$ and it should be worth finding DDAHA representation of the irregular conformal block.

Finally, the mixture of bulk and micro Coulomb charges in two dimensions is an interesting system whose interaction is represented in terms of the logarithmic potential. If the system is fine-tuned so that the system shows the conformal symmetry, then the matrix model should play the role. In addition, if the bulk charges are localized so that they are idealized in terms of finite number of multi-poles, then the free energy of the irregular matrix model can be useful.

\section{Acknowledgments}

This work is supported by the National Research Foundation of Korea (NRF) grant funded by the Korea government (MSIP) (NRF-2014R1A2A2A01004951). 


\section{A Loop equation of $A_{2}$ irregular matrix model}

The $A_{2}$ irregular matrix model (3.1) has the Virasoro and $W_{3}$ symmetry which is represented in terms of loop equations [36, 52-54]. We put the multi-point resolvent as

$$
R_{K_{1} ; \cdots ; K_{s}}\left(z_{1}, \cdots, z_{s}\right)=\beta\left(\frac{g}{\sqrt{\beta}}\right)^{2-s}\left\langle\sum_{i_{1}=1}^{N_{K_{1}}} \frac{1}{z_{1}-\lambda_{i_{1} ; K_{1}}} \cdots \sum_{i_{s}=1}^{N_{K_{s}}} \frac{1}{z_{s}-\lambda_{i_{s} ; K_{s}}}\right\rangle_{\text {connected }} .
$$

where denoting $\lambda_{i ; 1}=x_{i}, \lambda_{j ; 2}=y_{j}$. One obtains the quadratic loop equation if one performs the conformal transformation of the integration variables $x_{i} \rightarrow x_{i}+\varepsilon /\left(x_{i}-z\right)$ and $y_{j} \rightarrow y_{j}+\varepsilon /\left(y_{j}-z\right)$ which provides the Virasoro symmetry:

$$
\begin{aligned}
& R_{1}(z)^{2}+R_{2}(z)^{2}-R_{1}(z) R_{2}(z)+V_{1}^{\prime}(z) R_{1}(z)+V_{2}^{\prime}(z) R_{2}(z) \\
& \quad+\frac{\hbar Q}{2}\left(R_{1}^{\prime}(z)+R_{2}^{\prime}(z)\right)-\frac{\hbar^{2}}{4}\left(R_{1 ; 1}(z, z)-R_{1 ; 2}(z, z)+R_{2 ; 2}(z, z)\right)=\frac{f_{1}(z)+f_{2}(z)}{4},
\end{aligned}
$$

where $f_{1}(z):=4 g \sqrt{\beta} \sum_{i}^{N_{1}}\left\langle\frac{V_{1}^{\prime}(z)-V_{1}^{\prime}\left(x_{i}\right)}{z-x_{i}}\right\rangle$ and $f_{2}(z):=4 g \sqrt{\beta} \sum_{i}^{N_{2}}\left\langle\frac{V_{2}^{\prime}(z)-V_{2}^{\prime}\left(y_{j}\right)}{z-y_{j}}\right\rangle$. Here $\langle\cdots\rangle$ denotes the expectation value with respect to the $A_{2}$ matrix model.

$W_{3}$ symmetry is given in terms of cubic loop equation [36]

$$
\begin{aligned}
0= & -R_{1}^{2} R_{2}+R_{1} R_{2}^{2}-V_{1}^{\prime}\left(R_{1}^{2}+V_{1}^{\prime} R_{1}-\frac{f_{1}}{4}\right)+V_{2}^{\prime}\left(R_{2}^{2}+V_{2}^{\prime} R_{2}-\frac{f_{2}}{4}\right)+\frac{g_{1}-g_{2}}{4} \\
& +\frac{\hbar Q}{4}\left[3\left(V_{2}^{\prime} R_{2}^{\prime}-V_{1}^{\prime} R_{1}^{\prime}\right)+R_{1} R_{2}^{\prime}-R_{1}^{\prime} R_{2}+2\left(R_{2} R_{2}^{\prime}-R_{1} R_{1}^{\prime}\right)+V_{2}^{\prime \prime} R_{2}-V_{1}^{\prime \prime} R_{1}+\frac{f_{1}^{\prime}-f_{2}^{\prime}}{4}\right] \\
& +\frac{\hbar^{2} Q^{2}}{8}\left(R_{2}^{\prime \prime}-R_{1}^{\prime \prime}\right)+\frac{\hbar^{2}}{4}\left[V_{1}^{\prime} R_{1 ; 1}-V_{2}^{\prime} R_{2 ; 2}+R_{1 ; 1} R_{2}-R_{2 ; 2} R_{1}-2 R_{1 ; 2}\left(R_{2}-R_{1}\right)\right] \\
& +\frac{\hbar^{3} Q}{16}\left[R_{1 ; 1}^{\prime}-R_{2 ; 2}^{\prime}+\lim _{\bar{z} \rightarrow z}\left(\frac{\partial}{\partial z} R_{1 ; 2}(z, \bar{z})-\frac{\partial}{\partial \bar{z}} R_{1 ; 2}(z, \bar{z})\right)\right]+\frac{\hbar^{4}}{16}\left(R_{1 ; 2 ; 2}-R_{1 ; 1 ; 2}\right),
\end{aligned}
$$

where $g_{1}(z):=4 g^{2} \beta \sum_{i, j}\left\langle\frac{V_{1}^{\prime}(z)-V_{1}^{\prime}\left(x_{i}\right)}{\left(z-x_{i}\right)\left(x_{i}-y_{j}\right)}\right\rangle$ and $g_{2}(z):=4 g^{2} \beta \sum_{i, j}\left\langle\frac{V_{1}^{\prime}(z)-V_{2}^{\prime}\left(y_{j}\right)}{\left(z-y_{j}\right)\left(y_{j}-x_{i}\right)}\right\rangle$. This is obtained after varying the integration variables $x_{i} \rightarrow x_{i}+\sum_{j=1}^{N_{2}} \frac{\epsilon}{\left(x_{i}-z\right)\left(x_{i}-y_{j}\right)}$ and $y_{j} \rightarrow$ $y_{j}+\sum_{i=1}^{N_{1}} \frac{\epsilon}{\left(y_{j}-z\right)\left(x_{i}-y_{j}\right)}$.

At the classical/NS limit $(\hbar \rightarrow 0, b \rightarrow \infty$ while $\hbar b=\epsilon$ finite), each multi-point resolvent is finite but due to the factor $\hbar$, the multi-point resolvent terms drop out and the loop equations are given in the simple form:

$$
\begin{aligned}
X_{1}^{2}+X_{2}^{2}-X_{1} X_{2}+2 \epsilon\left(X_{1}^{\prime}+X_{2}^{\prime}\right) & =-\xi_{2}, \\
X_{1}^{2} X_{2}-X_{1} X_{2}^{2}+\frac{\epsilon}{2}\left[\left(2 X_{1}+X_{2}\right) X_{1}^{\prime}-\left(X_{1}+2 X_{2}\right) X_{2}^{\prime}\right]+\frac{\epsilon^{2}}{2}\left(X_{1}^{\prime \prime}-X_{2}^{\prime \prime}\right) & =\frac{2}{3 \sqrt{3}} \xi_{3},
\end{aligned}
$$


where $X_{1} / 2=R_{1}+\left(2 V_{1}^{\prime}+V_{2}^{\prime}\right) / 3, X_{2} / 2=R_{2}+\left(V_{1}^{\prime}+2 V_{2}^{\prime}\right) / 3$ and

$$
\begin{aligned}
\xi_{2}= & -2 \epsilon\left(V_{1}^{\prime \prime}+V_{2}^{\prime \prime}\right)-\frac{4}{3}\left[\left(V_{1}^{\prime}\right)^{2}+V_{1}^{\prime} V_{2}^{\prime}+\left(V_{2}^{\prime}\right)^{2}\right]-\left(f_{1}+f_{2}\right), \\
\xi_{3}= & \frac{4 \sqrt{3}}{9}\left(2\left(V_{1}^{\prime}\right)^{3}+3\left(V_{1}^{\prime}\right)^{2} V_{2}^{\prime}-3 V_{1}^{\prime}\left(V_{2}^{\prime}\right)^{2}-2\left(V_{2}^{\prime}\right)^{3}\right) \\
& +\sqrt{3} \epsilon\left(2 V_{1}^{\prime} V_{1}^{\prime \prime}+V_{2}^{\prime} V_{1}^{\prime \prime}-2 V_{2}^{\prime} V_{2}^{\prime \prime}-V_{1}^{\prime} V_{2}^{\prime \prime}\right)+\frac{\sqrt{3}}{2} \epsilon^{2}\left(V_{1}^{\prime \prime \prime}-V_{2}^{\prime \prime \prime}\right) \\
& +\sqrt{3}\left(\left(f_{1}-2 f_{2}\right) V_{1}^{\prime}+\left(2 f_{1}-f_{2}\right) V_{2}^{\prime}\right)+3 \sqrt{3}\left(g_{1}-g_{2}\right)+\frac{3 \sqrt{3}}{4} \epsilon\left(f_{1}^{\prime}-f_{2}^{\prime}\right),
\end{aligned}
$$

$\xi_{2}$ and $\xi_{3}$ look complicated but can be written in a compact form if one uses the mode expansion,

$$
\xi_{2}=\sum_{k=-2 m}^{2 n} \frac{A_{k}}{z^{k+2}}-\sum_{k=-m}^{n-1} \frac{d_{k}}{z^{k+2}}, \quad \xi_{3}=\sum_{k=-3 m}^{3 n} \frac{M_{k}}{z^{k+3}}-\sum_{k=-2 m}^{2 n-1} \frac{e_{k}}{z^{k+3}},
$$

where $A_{k}$ and $B_{k}$ are constants (here we use the notation $c_{\ell}$ which is related with $a_{\ell}=$ $\left.\left(\sqrt{3} c_{\ell}-b_{\ell}\right) / 2\right)$

$$
\begin{aligned}
A_{k}= & \epsilon(k+1)\left(b_{k}+\sqrt{3} c_{k}\right)-\sum_{r+s=k}\left(b_{r} b_{s}+c_{r} c_{s}\right), \\
B_{k}= & \sum_{r+s+t=k}\left(3 c_{r} b_{s} b_{t}-c_{r} c_{s} c_{t}\right)+\frac{3}{2} \epsilon \sum_{r+s=k}(s+1)\left(\sqrt{3} c_{r} c_{s}-2 c_{r} b_{s}-\sqrt{3} b_{r} b_{s}\right) \\
& +\frac{3}{4} \epsilon^{2}(k+1)(k+2)\left(\sqrt{3} b_{k}-c_{k}\right) .
\end{aligned}
$$

$d_{k}$ is the mode of $f_{1}+f_{2}=\sum_{k=-m}^{n-1} \frac{d_{k}}{z^{k+2}}$ and induces the flow equation when $-(m-1) \leq$ $k \leq n-1$;

$$
\begin{aligned}
& d_{k}=\left\{\begin{array}{lc}
v_{k}^{\partial}\left(-\hbar^{2} \log \mathcal{Z}_{(m ; n)}\right), & 0 \leq k \leq n-1 \\
2 \epsilon\left(b_{k} N_{b}+c_{k} N_{c}\right)+u_{k}^{\partial}\left(-\hbar^{2} \log \mathcal{Z}_{(m ; n)}\right), & -(m-1) \leq k \leq-1
\end{array}\right. \\
& v_{k}^{\partial}=\sum_{\substack{r-s=k \\
0<s}} s\left(b_{r} \frac{\partial}{\partial b_{s}}+c_{r} \frac{\partial}{\partial c_{s}}\right), \quad u_{k}^{\partial}=\sum_{\substack{r-s=k \\
s<0}}(-s)\left(b_{r} \frac{\partial}{\partial b_{s}}+c_{r} \frac{\partial}{\partial c_{s}}\right)
\end{aligned}
$$

where $N_{b}=N-M / 2, N_{c}=\sqrt{3} M / 2$. On the other hand, $d_{-m}$ is constant, $d_{-m}=$ $2 \epsilon\left(b_{-m} N_{b}+c_{-m} N_{c}\right)$.

The mode $e_{k}$ is defined as

$$
-\sum_{k=-2 m}^{2 n-1} \frac{e_{k}}{z^{k+3}}=\sqrt{3}\left(\left(f_{1}-2 f_{2}\right) V_{1}^{\prime}+\left(2 f_{1}-f_{2}\right) V_{2}^{\prime}\right)+3 \sqrt{3}\left(g_{1}-g_{2}\right)+\frac{3 \sqrt{3}}{4} \epsilon\left(f_{1}^{\prime}-f_{2}^{\prime}\right),
$$


and also induces the flow equation. When $-(2 m-1) \leq k \leq 2 n-1$, we have

$$
e_{k}= \begin{cases}-\frac{1}{\mathcal{Z}_{(m ; n)}} \hbar^{2} \mu_{k}^{\partial} \mathcal{Z}_{(m ; n)}, & n \leq k \leq 2 n-1 \\ -\left.\frac{1}{\mathcal{Z}_{(m ; 2 n-k)}}\left(\hbar^{2} \mu_{k}^{\partial}+\hbar^{4} \mu_{k}^{\partial^{2}}\right) \mathcal{Z}_{(m ; 2 n-k)}\right|_{\left\{b_{k>n}, c_{k>n}\right\} \rightarrow 0}, & 0 \leq k \leq n-1 \\ \nu_{k}^{c}-\left.\frac{1}{\mathcal{Z}_{(2 m+k ; n)}}\left(\hbar^{2} \nu_{k}^{\partial}+\hbar^{4} \nu_{k}^{\partial^{2}}\right) \mathcal{Z}_{(2 m+k ; n)}\right|_{\left\{b_{-k>m}, c_{-k>m}\right\} \rightarrow 0}, & -(m-1) \leq k \leq-1 \\ \nu_{k}^{c}-\frac{1}{\mathcal{Z}_{(m ; n)}} \hbar^{2} \nu_{k}^{\partial} \mathcal{Z}_{(m ; n)}, & -(2 m-1) \leq k \leq-m .\end{cases}
$$

where

$$
\begin{aligned}
& \mu_{k}^{\partial}=\sum_{\substack{r+s-t=k \\
t>0}} \frac{t}{2}\left(3 c_{r} c_{s} \frac{\partial}{\partial c_{t}}-6 c_{r} b_{s} \frac{\partial}{\partial b_{t}}-3 b_{r} b_{s} \frac{\partial}{\partial c_{t}}\right) \\
& -\frac{3}{2} \epsilon \sum_{\substack{r-s=k \\
s>0}} \frac{s}{2}\left[(1+r)\left(\sqrt{3} c_{r} \frac{\partial}{\partial c_{s}}-2 b_{r} \frac{\partial}{\partial c_{s}}-\sqrt{3} b_{r} \frac{\partial}{\partial b_{s}}\right)\right. \\
& \left.+(1-s)\left(\sqrt{3} c_{r} \frac{\partial}{\partial c_{s}}-2 c_{r} \frac{\partial}{\partial b_{s}}-\sqrt{3} b_{r} \frac{\partial}{\partial b_{s}}\right)\right], \\
& \mu_{k}^{\partial^{2}}=-\sum_{\substack{r-s-t=k, s, t>0}} \frac{s t}{4}\left(3 c_{r} \frac{\partial}{\partial c_{s}} \frac{\partial}{\partial c_{t}}-6 b_{r} \frac{\partial}{\partial b_{s}} \frac{\partial}{\partial c_{t}}-3 c_{r} \frac{\partial}{\partial b_{s}} \frac{\partial}{\partial b_{t}}\right), \\
& \nu_{k}^{\partial}=\sum_{\substack{r+s-t=k \\
t<0}} \frac{(-t)}{2}\left(3 c_{r} c_{s} \frac{\partial}{\partial c_{t}}-6 b_{r} c_{s} \frac{\partial}{\partial b_{t}}-3 b_{r} b_{s} \frac{\partial}{\partial c_{t}}\right) \\
& +\epsilon \sum_{\substack{r-s=k \\
s<0}} \frac{(-s)}{2}\left(6 c_{r} N_{c} \frac{\partial}{\partial c_{s}}-6 b_{r} N_{b} \frac{\partial}{\partial c_{s}}-6 b_{r} N_{c} \frac{\partial}{\partial b_{s}}-6 c_{r} N_{b} \frac{\partial}{\partial b_{s}}\right) \\
& -\frac{3}{2} \epsilon \sum_{\substack{r-s=k \\
s<0}} \frac{(-s)}{2}\left[(1+r)\left(\sqrt{3} c_{r} \frac{\partial}{\partial c_{s}}-2 b_{r} \frac{\partial}{\partial c_{s}}-\sqrt{3} b_{r} \frac{\partial}{\partial b_{s}}\right)\right. \\
& \left.+(1-s)\left(\sqrt{3} c_{r} \frac{\partial}{\partial c_{s}}-2 c_{r} \frac{\partial}{\partial b_{s}}-\sqrt{3} b_{r} \frac{\partial}{\partial b_{s}}\right)\right], \\
& \nu_{k}^{\partial^{2}}=-\sum_{\substack{r-s-t=k \\
s, t<0}} \frac{s t}{4}\left(3 c_{r} \frac{\partial}{\partial c_{s}} \frac{\partial}{\partial c_{t}}-6 b_{r} \frac{\partial}{\partial b_{s}} \frac{\partial}{\partial c_{t}}-3 c_{r} \frac{\partial}{\partial b_{s}} \frac{\partial}{\partial b_{t}}\right), \\
& \nu_{k}^{c}=+\epsilon \sum_{r+s=k}\left(3 N_{c} c_{r} c_{s}-6 N_{b} c_{r} b_{s}-3 N_{c} b_{r} b_{s}\right)+\epsilon^{2}\left(3 c_{k} N_{c}^{2}-6 b_{k} N_{b} N_{c}-3 c_{k} N_{b}^{2}\right) \\
& -\frac{3}{2} \epsilon^{2}\left[(k+1)\left(\sqrt{3} c_{k} N_{c}-2 b_{k} N_{c}-\sqrt{3} b_{k} N_{b}\right)+\left(\sqrt{3} c_{k} N_{c}-2 c_{k} N_{b}-\sqrt{3} b_{k} N_{b}\right)\right] .
\end{aligned}
$$

Note that the $\epsilon$ terms in $\mu_{k}^{\partial}$ vanished identically when $k \geq n$ and same for $\nu_{k}^{\partial}$ when $k \leq-m$. And $e_{-2 m}=\nu_{-2 m}^{c}$ is a constant. 
Note that we introduced the extended partition function in (A.10); $\mathcal{Z}_{(m ; 2 n-k)}$ for $0 \leq$ $k \leq n-1$ and $\mathcal{Z}_{(2 m+k ; n)}$ for $-(m-1) \leq k \leq-1$. This is because $g_{1}$ and $g_{2}$ can have the expectation values $\left\langle 1 / x_{i}^{r}\right\rangle,\left\langle 1 / y_{j}^{r}\right\rangle$ with $-(2 m+k) \leq r<-m$ and $n<r \leq 2 n-k$. To represent these expectation values in terms of derivatives of the partition function, we need to extend the parameter space up to $b_{2 n-k}, c_{2 n-k}$ when $0 \leq k \leq n-1$, and up to $b_{-(2 m+k)}$ and $c_{-(2 m+k)}$ when $-(m-1) \leq k \leq-1$. After evaluation of the derivatives, we put the parameters zero [36].

\section{B Representation of W3 currents}

$\xi_{2}$ and $\xi_{3}$ are the expectation values of the Virasoro and $\mathcal{W}_{3}$ current:

$$
\xi_{2}=\frac{\left\langle I_{m}|T(z)| I_{n}\right\rangle}{\left\langle I_{m} \mid I_{n}\right\rangle}, \quad \xi_{3}=\frac{\left\langle I_{m}|W(z)| I_{n}\right\rangle}{\left\langle I_{m} \mid I_{n}\right\rangle} .
$$

One can check that the modes of $\xi_{2}$ and $\xi_{3}$ in (A.8) are compatible with the $\mathcal{W}_{3}$ algebraic commutation relation:

$$
\begin{aligned}
{\left[L_{p}, L_{q}\right] } & =(p-q) L_{p+q}+\frac{c}{12}\left(p^{3}-p\right) \delta_{p,-q}, \\
{\left[L_{p}, W_{q}\right] } & =(2 p-q) W_{p+q}, \\
-\frac{2}{9}\left(\frac{32}{22+5 c}\right)\left[W_{p}, W_{q}\right] & =\frac{c}{3 \cdot 5 !}\left(p^{2}-1\right)\left(p^{2}-4\right) p \delta_{p,-q}+\frac{16}{22+5 c}(p-q) \Lambda_{p+q} \\
& +(p-q)\left(\frac{1}{15}(p+q+2)(p+q+3)-\frac{1}{6}(p+2)(q+2)\right) L_{p+q},
\end{aligned}
$$

where $^{2}$

$$
\begin{aligned}
\Lambda_{p} & =\sum_{k=-\infty}^{\infty}: L_{k} L_{p-k}:+\frac{1}{5} x_{p} L_{p}, \\
x_{2 \ell} & =(\ell+1)(\ell-1), \quad x_{2 \ell+1}=(2+\ell)(1-\ell),
\end{aligned}
$$

and the central charge $c=2+24 \epsilon^{2}$.

Note that the negative generators $L_{-k}$ and $W_{-k}(k>0)$ obtained in (A.9) and (A.10) are left representation in the sense that negative generators should act on the bra $\left\langle I_{m}\right|$. However, to check the commutation relation (B.4) we need to find right representations of negative generators acting on ket $\left|I_{n}\right\rangle$. To find the right representation we follow the trick used in [36]: use the transformation of the integration variables $x_{i} \rightarrow x_{i}+\varepsilon / x_{i}^{r}$ and $y_{j} \rightarrow y_{j}+\varepsilon / y_{j}^{r}$ to obtain two identities which can be used to find the relation for $d_{k}$ $(k \leq-1)$ :

$$
d_{k}=-\left.\frac{\left(\hbar^{2} v_{k}^{\partial}+\hbar^{4} v_{k}^{\partial^{2}}\right) \mathcal{Z}_{(m ; n-k)}}{\mathcal{Z}_{(m ; n-k)}}\right|_{\left\{b_{k>n}, c_{k>n}\right\} \rightarrow 0},
$$

\footnotetext{
${ }^{2}$ If one rescales $W_{p}$ as $i \frac{3}{\sqrt{2}} \sqrt{\frac{22+5 c}{32}} W_{p}$, then the algebra reduces to the original Fateev and Zamolodchikov convention [55]. Note that because of the factor $i$, the modes of $W(z)$ are anti-hermitian: $W_{k}^{\dagger}=-W_{k}$.
} 
where

$$
v_{k}^{\partial^{2}}=-\sum_{-(r+s)=k} \frac{r s}{4}\left(\frac{\partial}{\partial b_{r}} \frac{\partial}{\partial b_{s}}+\frac{\partial}{\partial c_{r}} \frac{\partial}{\partial c_{s}}\right)+\frac{\epsilon}{2} k(k+1)\left(\frac{\partial}{\partial b_{-k}}+\sqrt{3} \frac{\partial}{\partial c_{-k}}\right),
$$

When $k<-m$, one realizes that $d_{k}$ vanishes identically and $v_{-1}^{\partial^{2}}=0$ by definition.

Likewise, after the change of variables $x_{i} \rightarrow x_{i}+\sum_{j=1}^{N_{2}} \frac{\varepsilon}{\left(x_{i}-y_{j}\right) x_{i}^{r}}$ and $y_{j} \rightarrow y_{j}+$ $\sum_{i=1}^{N_{1}} \frac{\varepsilon}{\left(x_{i}-y_{j}\right) y_{j}^{r}}$, one finds the right representation of the negative mode $k \leq-1 \mathcal{W}_{3}$ current:

$$
e_{k}=-\left.\frac{\left(\hbar^{2} \mu_{k}^{\partial}+\hbar^{4} \mu_{k}^{\partial^{2}}+\hbar^{6} \mu_{k}^{\partial^{3}}\right) \mathcal{Z}_{(m ; 2 n-k)}}{\mathcal{Z}_{(m ; 2 n-k)}}\right|_{\left\{b_{k>n}, c_{k>n}\right\} \rightarrow 0},
$$

where

$$
\begin{aligned}
\mu_{k}^{\partial^{3}}= & -\sum_{-(r+s+t)=k} \frac{r s t}{8}\left(3 \frac{\partial}{\partial b_{r}} \frac{\partial}{\partial b_{s}} \frac{\partial}{\partial b_{t}}-\frac{\partial}{\partial c_{r}} \frac{\partial}{\partial c_{s}} \frac{\partial}{\partial c_{t}}\right) \\
& +\frac{3}{2} \epsilon \sum_{-(r+s)=k} \frac{r s(1-s)}{4}\left(\sqrt{3} \frac{\partial}{\partial c_{r}} \frac{\partial}{\partial c_{s}}-2 \frac{\partial}{\partial c_{r}} \frac{\partial}{\partial b_{s}}-\sqrt{3} \frac{\partial}{\partial b_{r}} \frac{\partial}{\partial b_{s}}\right) \\
& +\frac{3}{8} \epsilon^{2} k(k+1)(k+2)\left(\sqrt{3} \frac{\partial}{\partial b_{-k}}-\frac{\partial}{\partial c_{-k}}\right) .
\end{aligned}
$$

We have $e_{k<-2 m}=0$ and $\mu_{k}^{\partial^{3}}=0$ for $k=-1,-2$.

If we define the differential operator $v_{k}$ and $\mu_{k}$ by

$$
v_{k}=\left\{\begin{array}{ll}
v_{k}^{\partial}, & -1 \leq k \leq n-1 \\
v_{k}^{\partial}+v_{k}^{\partial^{2}}, & k \leq-2
\end{array} \quad, \quad \mu_{k}= \begin{cases}\mu_{k}^{\partial}, & n \leq k \leq 2 n-1 \\
\mu_{k}^{\partial}+\mu_{k}^{\partial^{2}}, & -2 \leq k \leq n-1 \\
\mu_{k}^{\partial}+\mu_{k}^{\partial^{2}}+\mu_{k}^{\partial^{3}}, & k \leq-3 .\end{cases}\right.
$$

then, the right representation of the Virasoro and $\mathcal{W}_{3}$ currents has the expression

$$
\mathcal{L}_{k}=\left\{\begin{array}{ll}
0, & 2 n<k \\
A_{k}, & n \leq k \leq 2 n \\
A_{k}+v_{k}, & -2 m \leq k \leq n-1 \\
v_{k}, & k<-2 m
\end{array} \quad, \quad \Omega_{k}= \begin{cases}0, & 3 n<k \\
M_{k}, & 2 n \leq k \leq 3 n \\
M_{k}+\mu_{k},-3 m \leq k \leq 2 n-1 \\
\mu_{k}, & k<-3 m .\end{cases}\right.
$$

where $\left\langle I_{m}\left|L_{k}\right| I_{n}\right\rangle:=\mathcal{L}_{k}\left\langle I_{m} \mid I_{n}\right\rangle$ and $\left\langle I_{m}\left|W_{k}\right| I_{n}\right\rangle:=\Omega_{k}\left\langle I_{m} \mid I_{n}\right\rangle$. One can check that the right representation satisfies the commutation relations (B.4).

\section{Perturbation method to find flow equations in $\boldsymbol{A}_{2}$ model}

In this section, we apply another method to find flow equations, with no need to assume $\epsilon$ to be small. Instead, we will suppose hierarchy in the Toda momentum $a_{k}$ and $b_{k}$. 


\section{C.1 $\mathcal{Z}_{(0 ; 1)}$}

Expanding in terms of $z$, to the highest power of (3.18), $z^{N+M-2}$ shows that

$$
d_{0}=\epsilon^{2}(N(N-1)-N M+M(M-1))+2 \epsilon\left[N b_{0}+M a_{0}\right],
$$

Expanding (3.19) in terms of $z$, for $z^{N-3-k}$ we have

$$
\begin{aligned}
0= & \epsilon^{3} P_{N-k}(N-k)(N-k-1)(N-k-2) \\
& +2 \epsilon^{2} \sum_{t=-m}^{n} P_{N-k+t}(N-k+t)(N-k-2)\left(2 b_{t}+a_{t}\right) \\
& +\epsilon\left(\sum_{t=-2 m}^{2 n} P_{N-k+t}(N-k+t) A_{t}-\sum_{t=-m}^{n-1} P_{N-k+t}(N-k+t) d_{t}\right. \\
& \left.\quad+\frac{4}{3} \sum_{t=-2 m}^{2 n} P_{N-k+t}(N-k+t)\left[\sum_{s=-m}^{n}\left(2 b_{s}+a_{s}\right)\left(2 b_{t-s}+a_{t-s}\right)\right]\right) \\
& -\sum_{t=-2 m}^{2 n-1} P_{N-k+t}\left[-\frac{2}{3 \sqrt{3}} e_{t}+\frac{2}{3} \sum_{s=-m}^{n-1} d_{s}\left(2 b_{t-s}+a_{t-s}\right)\right]+\frac{\epsilon}{2} \sum_{t=-m}^{n-1} P_{N-k+t}(t+2) d_{t} .
\end{aligned}
$$

The next power $z^{N+M-3}$ of (3.18) gives

$$
\begin{aligned}
& P_{N-1}\left\{\epsilon^{2}[M-2(N-1)]-2 \epsilon b_{0}\right\}+Q_{M-1}\left\{\epsilon^{2}[N-2(M-1)]-2 \epsilon a_{0}\right\} \\
& \quad=-2 \epsilon\left[N b_{1}+M a_{1}\right]
\end{aligned}
$$

Then let's turn back to eq. (3.19). For $z^{N-3-k}$,

$$
\begin{aligned}
0= & P_{N-k}\left\{-\left[-\frac{2}{3 \sqrt{3}} e_{0}+\frac{2}{3} d_{0}\left(2 b_{0}+a_{0}\right)-\epsilon d_{0}\right]+\epsilon^{3}(N-k)(N-k-1)(N-k-2)\right. \\
& \left.+2 \epsilon^{2}(N-k)(N-k-2)\left(2 b_{0}+a_{0}\right)+\epsilon(N-k)\left[-d_{0}+2 \epsilon\left(b_{0}+a_{0}\right)+4\left(b_{0}^{2}+a_{0} b_{0}\right)\right]\right\} \\
& +P_{N-k+1}\left\{-\left[-\frac{2}{3 \sqrt{3}} e_{1}+\frac{2}{3} d_{0}\left(2 b_{1}+a_{1}\right)\right]\right. \\
& \left.+2 \epsilon^{2}(N-k+1)(N-k-2)\left(2 b_{1}+a_{1}\right)+4 \epsilon(N-k+1)\left[\epsilon\left(b_{1}+a_{1}\right)+\left(2 b_{0} b_{1}+a_{1} b_{0}+a_{0} b_{1}\right)\right]\right\} \\
& +P_{N-k+2}\left\{\epsilon(N-k+2)\left[4\left(b_{1}^{2}+a_{1} b_{1}\right)\right]\right\},
\end{aligned}
$$

where we have used the definition of $A_{k}$ in (3.8). The corresponding equations of $Q_{M-k}$ can be obtained by setting $P_{N-k} \rightarrow Q_{M-k}, e_{k} \rightarrow-e_{k}$ and $b_{k} \rightarrow a_{k}, a_{k} \rightarrow b_{k}$.

At each power of $\mathrm{z}$, we have identities,

$$
\begin{gathered}
z^{N-3}:\left[-\frac{2}{3 \sqrt{3}} e_{0}+\frac{2}{3} d_{0}\left(2 b_{0}+a_{0}\right)-\epsilon d_{0}\right]= \\
\epsilon^{3} N(N-1)(N-2)+2 \epsilon^{2} N(N-2)\left(2 b_{0}+a_{0}\right) \\
\quad+\epsilon N\left[-d_{0}+2 \epsilon\left(b_{0}+a_{0}\right)+4\left(b_{0}^{2}+a_{0} b_{0}\right)\right]
\end{gathered}
$$




$$
\begin{aligned}
z^{N-4}: \quad 0= & P_{N-1}\left\{-\left[-\frac{2}{3 \sqrt{3}} e_{0}+\frac{2}{3} d_{0}\left(2 b_{0}+a_{0}\right)-\epsilon d_{0}\right]+\epsilon^{3}(N-1)(N-2)(N-3)\right. \\
& \left.+2 \epsilon^{2}(N-1)(N-3)\left(2 b_{0}+a_{0}\right)+\epsilon(N-1)\left[-d_{0}+2 \epsilon\left(b_{0}+a_{0}\right)+4\left(b_{0}^{2}+a_{0} b_{0}\right)\right]\right\} \\
& +\left\{-\left[-\frac{2}{3 \sqrt{3}} e_{1}+\frac{2}{3} d_{0}\left(2 b_{1}+a_{1}\right)\right]\right. \\
& \left.+2 \epsilon^{2} N(N-3)\left(2 b_{1}+a_{1}\right)+4 \epsilon N\left[\epsilon\left(b_{1}+a_{1}\right)+\left(2 b_{0} b_{1}+a_{1} b_{0}+a_{0} b_{1}\right)\right]\right\}, \\
z^{N-5}: \quad 0= & P_{N-2}\left\{-\left[-\frac{2}{3 \sqrt{3}} e_{0}+\frac{2}{3} d_{0}\left(2 b_{0}+a_{0}\right)-\epsilon d_{0}\right]+\epsilon^{3}(N-2)(N-3)(N-4)\right. \\
& \left.+2 \epsilon^{2}(N-2)(N-4)\left(2 b_{0}+a_{0}\right)+\epsilon(N-2)\left[-d_{0}+2 \epsilon\left(b_{0}+a_{0}\right)+4\left(b_{0}^{2}+a_{0} b_{0}\right)\right]\right\} \\
& +P_{N-1}\left\{-\left[-\frac{2}{3 \sqrt{3}} e_{1}+\frac{2}{3} d_{0}\left(2 b_{1}+a_{1}\right)\right]\right. \\
& \left.+2 \epsilon^{2}(N-1)(N-4)\left(2 b_{1}+a_{1}\right)+4 \epsilon(N-1)\left[\epsilon\left(b_{1}+a_{1}\right)+\left(2 b_{0} b_{1}+a_{1} b_{0}+a_{0} b_{1}\right)\right]\right\} \\
& +\left\{\epsilon N\left[4\left(b_{1}^{2}+a_{1} b_{1}\right)\right]\right\},
\end{aligned}
$$

To find $e_{1}$, we use perturbation assuming $\left|b_{1}\right| \ll\left|a_{1}\right|$ so that $\left|P_{N-k}\right| \sim\left|b_{1}\right|^{k}$. Then at the first order we have from the above equations (C.6) and (C.7):

$$
\begin{aligned}
z^{N-4}: \quad \frac{2}{3 \sqrt{3}} e_{1}^{(1)}= & \frac{2}{3} d_{0} a_{1}-2 \epsilon^{2} N(N-1) a_{1}-4 \epsilon N b_{0} a_{1} \equiv B_{1}\left(b_{0}, a_{0}\right) a_{1}, \\
z^{N-5}: & =P_{N-1}^{(1)}\left\{-\left[-\frac{2}{3 \sqrt{3}} e_{1}^{(1)}+\frac{2}{3} d_{0} a_{1}\right]\right. \\
& \left.+2 \epsilon^{2}(N-1)(N-4) a_{1}+4 \epsilon(N-1)\left[\epsilon a_{1}+a_{1} b_{0}\right]\right\}+\left\{\epsilon N\left[4 a_{1} b_{1}\right]\right\}, \\
P_{N-1}^{(1)} & =\frac{N b_{1}}{\epsilon(N-1)+b_{0}} .
\end{aligned}
$$

At the second order, we have

$$
\begin{aligned}
z^{N-4}: \quad & \quad=P_{N-1}^{(1)}\left\{-\left[-\frac{2}{3 \sqrt{3}} e_{0}+\frac{2}{3} d_{0}\left(2 b_{0}+a_{0}\right)-\epsilon d_{0}\right]+\epsilon^{3}(N-1)(N-2)(N-3)\right. \\
& \left.+2 \epsilon^{2}(N-1)(N-3)\left(2 b_{0}+a_{0}\right)+\epsilon(N-1)\left[-d_{0}+2 \epsilon\left(b_{0}+a_{0}\right)+4\left(b_{0}^{2}+a_{0} b_{0}\right)\right]\right\} \\
& +\left\{-\left[-\frac{2}{3 \sqrt{3}} e_{1}^{(2)}+\frac{4}{3} d_{0} b_{1}\right]+4 \epsilon^{2} N(N-3) b_{1}+4 \epsilon N\left[\epsilon b_{1}+\left(2 b_{0} b_{1}+a_{0} b_{1}\right)\right]\right\},
\end{aligned}
$$

$$
\begin{aligned}
\frac{2}{3 \sqrt{3}} e_{1}^{(2)}= & \frac{4}{3} d_{0} b_{1}-4 \epsilon N\left(2 b_{0}+a_{0}\right) b_{1}-4 \epsilon^{2} N(N-2) b_{1}+\frac{N b_{1}}{\epsilon(N-1)+b_{0}}\left\{4 \epsilon\left(b_{0}+a_{0}\right) b_{0}\right. \\
& \left.+2 \epsilon^{2}\left(b_{0}+a_{0}\right)+2 \epsilon^{2}(2 N-3)\left(2 b_{0}+a_{0}\right)+3 \epsilon^{3}(N-1)(N-2)-\epsilon d_{0}\right\}(\text { C. } 12) \\
\equiv & B_{2}\left(b_{0}, a_{0}\right) b_{1} .
\end{aligned}
$$


Up to $\mathcal{O}\left(b_{1}\right)$ we find $e_{1}=e_{1}^{(1)}+e_{1}^{(2)}$. If one expands $e_{1}$ in terms of $\epsilon$, it reads

$$
\begin{aligned}
\frac{2}{3 \sqrt{3}} e_{1}= & \frac{4}{3} \epsilon\left[\left(a_{1}+2 b_{1}\right)\left(a_{0} M+b_{0} N\right)-3 b_{0} N\left(a_{1}+b_{1}\right)\right] \\
& +\epsilon^{2}\left[\frac{2}{3}\left(a_{1}+2 b_{1}\right)(N(N-1)+M(M-1)-N M)-2 N(N-1)\left(a_{1}+b_{1}\right)-2 N M \frac{a_{0}}{b_{0}} b_{1}\right] \\
& +\mathcal{O}\left(\epsilon^{3}\right) .
\end{aligned}
$$

This result is in perfect agreement with (3.28), expanding up to $\mathcal{O}\left(b_{1}\right)$. In fact, the perturbative condition $\left|b_{1}\right| \ll\left|a_{1}\right|$ is equivalent to choosing the filling fraction $N_{1}=N$, and $N_{2}=0$.

In this way we can find that

$$
\begin{aligned}
e_{1} & =e_{1}^{(1)}+e_{1}^{(2)}+e_{1}^{(3)}+\ldots+e_{1}^{(k+2)}+\ldots \\
& =B_{1}\left(b_{0}, a_{0}\right) a_{1}+B_{2}\left(b_{0}, a_{0}\right) b_{1}+B_{3}\left(b_{0}, a_{0}\right) b_{1} \frac{b_{1}}{a_{1}}+\ldots+B_{k+2}\left(b_{0}, a_{0}\right) b_{1}\left(\frac{b_{1}}{a_{1}}\right)^{k}+\ldots
\end{aligned}
$$

The flow equations for rank 1 case are

$$
-\hbar^{2} v_{0} \log \mathcal{Z}_{1}=d_{0}, \quad-\hbar^{2} \mu_{1} \log \mathcal{Z}_{1}=e_{1}
$$

where $v_{0}=b_{1} \frac{\partial}{\partial b_{1}}+a_{1} \frac{\partial}{\partial a_{1}}$ and $\mu_{1}=\sqrt{3}\left(a_{1}^{2}+2 a_{1} b_{1}\right) \frac{\partial}{\partial a_{1}}-\sqrt{3}\left(2 a_{1} b_{1}+b_{1}^{2}\right) \frac{\partial}{\partial b_{1}}$. From the first equation of (C.15) we find

$$
\hbar^{2} \log \mathcal{Z}_{1}=-d_{0} \log a_{1}+H(t),
$$

where $t:=b_{1} / a_{1}$ and $H(t)$ is a homogeneous solution to $v_{0}$. Put $H(t)$ into the second equation of (C.15), we get

$$
3(t+1) t \frac{\partial H(t)}{\partial t}=\frac{1}{\sqrt{3}} \frac{e_{1}}{a_{1}}-(1+2 t) d_{0} .
$$

From (C.14), it is clear that

$$
\frac{e_{1}}{a_{1}}=B_{1}\left(b_{0}, a_{0}\right)+B_{2}\left(b_{0}, a_{0}\right) t+B_{3}\left(b_{0}, a_{0}\right) t^{2}+\ldots
$$

Therefore, we have

$$
H(t)=\frac{1}{3}\left(\frac{B_{1}}{\sqrt{3}}-d_{0}\right) \log t-\frac{1}{3}\left(\frac{B_{1}-B_{2}+B_{3}}{\sqrt{3}}+d_{0}\right) \log (t+1)+\frac{B_{3}}{3 \sqrt{3}} t+\ldots,
$$

and partition function

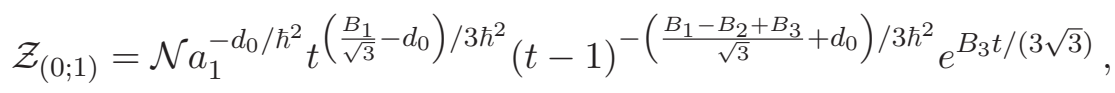

where $\mathcal{N}$ is a function of $a_{0}, b_{0}$ and $B_{k}$ with $k \geq 4$. 


\section{C.2 $\mathcal{Z}_{(0 ; 2)}$}

We need $d_{0}, d_{1}, e_{2}$ and $e_{3}$ to obtain the partition function. From the highest power $z^{N+M-2}$ of the quadratic equation (3.16) we obtain the expression of $d_{0}$ for any rank n. Now for rank 2 case, from the second highest power $z^{N+M-3}$ we have

$$
\begin{gathered}
P_{N-1}\left\{-d_{0}+\epsilon^{2}[(N-1)(N-2)-(N-1) M+(M-1) M]+2 \epsilon\left[(N-1) b_{0}+M a_{0}\right]\right\} \\
+Q_{M-1}\left\{-d_{0}+\epsilon^{2}[(M-1)(M-2)-(M-1) N+(N-1) N]+2 \epsilon\left[N b_{0}+(M-1) a_{0}\right]\right\} \\
\quad=d_{1}-2 \epsilon\left[N b_{1}+M a_{1}\right],
\end{gathered}
$$

From the cubic equation (3.19), we have

$$
\begin{aligned}
& z^{N-3}: \quad\left[-\frac{2}{3 \sqrt{3}} e_{0}+\frac{2}{3} d_{0}\left(2 b_{0}+a_{0}\right)-\epsilon d_{0}\right] \\
& =\epsilon^{3} N(N-1)(N-2)+2 \epsilon^{2} N(N-2)\left(2 b_{0}+a_{0}\right)+\epsilon N\left(A_{0}-d_{0}\right)+\frac{4}{3} \epsilon N\left(2 b_{0}+a_{0}\right)^{2}, \\
& z^{N-4}: \quad P_{N-1}\left[-\frac{2}{3 \sqrt{3}} e_{0}+\frac{2}{3} d_{0}\left(2 b_{0}+a_{0}\right)-\epsilon d_{0}\right] \\
& +\left[-\frac{2}{3 \sqrt{3}} e_{1}+\frac{2}{3} d_{0}\left(2 b_{1}+a_{1}\right)+\frac{2}{3} d_{1}\left(2 b_{0}+a_{0}\right)-\frac{3}{2} \epsilon d_{1}\right] \\
& =\epsilon^{3} P_{N-1}(N-1)(N-2)(N-3) \\
& +2 \epsilon^{2}\left(P_{N-1}(N-1)(N-3)\left(2 b_{0}+a_{0}\right)+N(N-3)\left(2 b_{1}+a_{1}\right)\right) \\
& +\epsilon\left(P_{N-1}(N-1)\left(A_{0}-d_{0}\right)+N\left(A_{1}-d_{1}\right)\right) \\
& +\frac{4}{3} \epsilon\left(P_{N-1}(N-1)\left(2 b_{0}+a_{0}\right)^{2}+2 N\left(2 b_{0}+a_{0}\right)\left(2 b_{1}+a_{1}\right)\right), \\
& z^{N-5}: \quad P_{N-2}\left[-\frac{2}{3 \sqrt{3}} e_{0}+\frac{2}{3} d_{0}\left(2 b_{0}+a_{0}\right)-\epsilon d_{0}\right] \\
& +P_{N-1}\left[-\frac{2}{3 \sqrt{3}} e_{1}+\frac{2}{3} d_{0}\left(2 b_{1}+a_{1}\right)+\frac{2}{3} d_{1}\left(2 b_{0}+a_{0}\right)-\frac{3}{2} \epsilon d_{1}\right] \\
& +\left[-\frac{2}{3 \sqrt{3}} e_{2}+\frac{2}{3} d_{0}\left(2 b_{2}+a_{2}\right)+\frac{2}{3} d_{1}\left(2 b_{1}+a_{1}\right)\right] \\
& =\epsilon^{3} P_{N-2}(N-2)(N-3)(N-4) \\
& +2 \epsilon^{2}\left(P_{N-2}(N-2)(N-4)\left(2 b_{0}+a_{0}\right)+P_{N-1}(N-1)(N-4)\left(2 b_{1}+a_{1}\right)\right. \\
& \left.+N(N-4)\left(2 b_{2}+a_{2}\right)\right) \\
& +\epsilon\left(P_{N-2}(N-2)\left(A_{0}-d_{0}\right)+P_{N-1}(N-1)\left(A_{1}-d_{1}\right)+N A_{2}\right) \\
& +\frac{4}{3} \epsilon\left(P_{N-2}(N-2)\left(2 b_{0}+a_{0}\right)^{2}+2 P_{N-1}(N-1)\left(2 b_{0}+a_{0}\right)\left(2 b_{1}+a_{1}\right)\right. \\
& \left.+N\left[2\left(2 b_{0}+a_{0}\right)\left(2 b_{2}+a_{2}\right)+\left(2 b_{1}+a_{1}\right)^{2}\right]\right),
\end{aligned}
$$




$$
\begin{aligned}
& z^{N-6}: \quad P_{N-3}\left[-\frac{2}{3 \sqrt{3}} e_{0}+\frac{2}{3} d_{0}\left(2 b_{0}+a_{0}\right)-\epsilon d_{0}\right] \\
& +P_{N-2}\left[-\frac{2}{3 \sqrt{3}} e_{1}+\frac{2}{3} d_{0}\left(2 b_{1}+a_{1}\right)+\frac{2}{3} d_{1}\left(2 b_{0}+a_{0}\right)-\frac{3}{2} \epsilon d_{1}\right] \\
& +P_{N-1}\left[-\frac{2}{3 \sqrt{3}} e_{2}+\frac{2}{3} d_{0}\left(2 b_{2}+a_{2}\right)+\frac{2}{3} d_{1}\left(2 b_{1}+a_{1}\right)\right] \\
& +\left[-\frac{2}{3 \sqrt{3}} e_{3}+\frac{2}{3} d_{1}\left(2 b_{2}+a_{2}\right)\right] \\
& =\epsilon^{3} P_{N-3}(N-3)(N-4)(N-5) \\
& +2 \epsilon^{2}\left(P_{N-3}(N-3)(N-5)\left(2 b_{0}+a_{0}\right)+P_{N-2}(N-2)(N-5)\left(2 b_{1}+a_{1}\right)\right. \\
& \left.+P_{N-1}(N-1)(N-5)\left(2 b_{2}+a_{2}\right)\right) \\
& +\epsilon\left(P_{N-3}(N-3)\left(A_{0}-d_{0}\right)+P_{N-2}(N-2)\left(A_{1}-d_{1}\right)+P_{N-1}(N-1) A_{2}\right. \\
& \left.+4 N\left(2 b_{1} b_{2}+a_{1} b_{2}+a_{2} b_{1}\right)\right) \\
& +\frac{4}{3} \epsilon\left(P_{N-3}(N-3)\left(2 b_{0}+a_{0}\right)^{2}+2 P_{N-2}(N-2)\left(2 b_{0}+a_{0}\right)\left(2 b_{1}+a_{1}\right)\right. \\
& \left.+P_{N-1}(N-1)\left[2\left(2 b_{0}+a_{0}\right)\left(2 b_{2}+a_{2}\right)+\left(2 b_{1}+a_{1}\right)^{2}\right]\right), \\
& z^{N-7}: \quad P_{N-4}\left[-\frac{2}{3 \sqrt{3}} e_{0}+\frac{2}{3} d_{0}\left(2 b_{0}+a_{0}\right)-\epsilon d_{0}\right] \\
& +P_{N-3}\left[-\frac{2}{3 \sqrt{3}} e_{1}+\frac{2}{3} d_{0}\left(2 b_{1}+a_{1}\right)+\frac{2}{3} d_{1}\left(2 b_{0}+a_{0}\right)-\frac{3}{2} \epsilon d_{1}\right] \\
& +P_{N-2}\left[-\frac{2}{3 \sqrt{3}} e_{2}+\frac{2}{3} d_{0}\left(2 b_{2}+a_{2}\right)+\frac{2}{3} d_{1}\left(2 b_{1}+a_{1}\right)\right] \\
& +P_{N-1}\left[-\frac{2}{3 \sqrt{3}} e_{3}+\frac{2}{3} d_{1}\left(2 b_{2}+a_{2}\right)\right] \\
& =\epsilon^{3} P_{N-4}(N-4)(N-5)(N-6) \\
& +2 \epsilon^{2}\left(P_{N-4}(N-4)(N-6)\left(2 b_{0}+a_{0}\right)+P_{N-3}(N-3)(N-6)\left(2 b_{1}+a_{1}\right)\right. \\
& \left.+P_{N-2}(N-2)(N-6)\left(2 b_{2}+a_{2}\right)\right) \\
& +\epsilon\left(P_{N-4}(N-4)\left(A_{0}-d_{0}\right)+P_{N-3}(N-3)\left(A_{1}-d_{1}\right)+P_{N-2}(N-2) A_{2}\right. \\
& \left.+P_{N-1}(N-1) A_{3}+4 N b_{2}\left(b_{2}+a_{2}\right)\right) \\
& +\frac{4}{3} \epsilon\left(P_{N-4}(N-4)\left(2 b_{0}+a_{0}\right)^{2}+2 P_{N-3}(N-3)\left(2 b_{0}+a_{0}\right)\left(2 b_{1}+a_{1}\right)\right. \\
& +P_{N-2}(N-2)\left[2\left(2 b_{0}+a_{0}\right)\left(2 b_{2}+a_{2}\right)+\left(2 b_{1}+a_{1}\right)^{2}\right] \\
& \left.+2 P_{N-1}(N-1)\left(2 b_{1}+a_{1}\right)\left(2 b_{2}+a_{2}\right)\right) \text {. }
\end{aligned}
$$

Perturbation holds if we require $\left|b_{2} / b_{1}\right| \ll\left|b_{1}\right| \ll 1,\left|b_{2}\right| \ll\left|a_{2}\right|$, and $\left|b_{1}\right| \sim\left|a_{1}\right|$, so that 
$\left|P_{N-k}\right| \sim\left|b_{2} / b_{1}\right|^{k}$ is ensured. Then at the first order of the perturbation, we have: from the quadratic equation:

$$
\begin{aligned}
d_{0} & =\epsilon^{2}(N(N-1)-N M+M(M-1))+2 \epsilon\left[N b_{0}+M a_{0}\right], \\
z^{N+M-3}: \quad d_{1}^{(1)} & =2 \epsilon\left[N b_{1}+M a_{1}\right] .
\end{aligned}
$$

From the equations (C.23) to (C.26):

$$
\begin{array}{ll}
z^{N-4}: \quad & {\left[-\frac{2}{3 \sqrt{3}} e_{1}^{(1)}+\frac{2}{3} d_{0}\left(2 b_{1}+a_{1}\right)+\frac{2}{3} d_{1}^{(1)}\left(2 b_{0}+a_{0}\right)-\frac{3}{2} \epsilon d_{1}^{(1)}\right]} \\
& =2 \epsilon^{2} N(N-3)\left(2 b_{1}+a_{1}\right)+\epsilon N\left(A_{1}-d_{1}^{(1)}\right)+\frac{8}{3} \epsilon N\left(2 b_{0}+a_{0}\right)\left(2 b_{1}+a_{1}\right), \\
z^{N-5}: \quad & {\left[-\frac{2}{3 \sqrt{3}} e_{2}^{(1)}+\frac{2}{3} d_{0} a_{2}+\frac{2}{3} d_{1}^{(1)}\left(2 b_{1}+a_{1}\right)\right]} \\
& =2 \epsilon^{2} N(N-4) a_{2}+\epsilon N A_{2}+\frac{4}{3} \epsilon N\left[2\left(2 b_{0}+a_{0}\right) a_{2}+\left(2 b_{1}+a_{1}\right)^{2}\right], \\
z^{N-6}: \quad & {\left[-\frac{2}{3 \sqrt{3}} e_{3}^{(1)}+\frac{2}{3} d_{1}^{(1)} a_{2}\right]=4 \epsilon N a_{2} b_{1},} \\
z^{N-7}: \quad & P_{N-1}^{(1)}\left[-\frac{2}{3 \sqrt{3}} e_{3}^{(1)}+\frac{2}{3} d_{1}^{(1)} a_{2}\right] \\
= & \epsilon\left(P_{N-1}^{(1)}(N-1) A_{3}+4 N b_{2} a_{2}\right)+\frac{8}{3} \epsilon P_{N-1}(N-1)\left(2 b_{1}+a_{1}\right) a_{2} .
\end{array}
$$

Thus $d_{0}, d_{1}, e_{2}$ and $e_{3}$ are obtained.

\section{C.3 $\mathcal{Z}_{(1 ; 1)}$}

We need $d_{0}, e_{-1}$ and $e_{1}$ to evaluate. From the power expansion of the quadratic equation, we know

$$
\begin{aligned}
z^{N+M-1}: \quad d_{-1}= & 2 \epsilon\left[N b_{-1}+M a_{-1}\right], \\
z^{N+M-2}: \quad d_{-1} & \left(P_{N-1}+Q_{M-1}\right)+d_{0} \\
= & \epsilon^{2}(N(N-1)-N M+M(M-1))+2 \epsilon\left[N b_{0}+M a_{0}\right] \\
& +2 \epsilon\left[b_{-1}\left((N-1) P_{N-1}+N Q_{M-1}\right)+a_{-1}\left((M-1) Q_{M-1}+M P_{N-1}\right)\right], \\
z^{N+M-3}: \quad d_{-1} & \left(P_{N-2}+Q_{M-2}+P_{N-1} Q_{M-1}\right)+d_{0}\left(P_{N-1}+Q_{M-1}\right) \\
= & P_{N-1}\left\{\epsilon^{2}[(N-1)(N-2)-(N-1) M+(M-1) M]+2 \epsilon\left[(N-1) b_{0}+M a_{0}\right]\right\} \\
& +Q_{M-1}\left\{\epsilon^{2}[(M-1)(M-2)-(M-1) N+(N-1) N]+2 \epsilon\left[N b_{0}+(M-1) a_{0}\right]\right\} \\
& +2 \epsilon\left[b_{-1}\left((N-2) P_{N-2}+N Q_{M-2}+(N-1) P_{N-1} Q_{M-1}\right)\right. \\
& \left.+a_{-1}\left((M-2) Q_{M-2}+M P_{N-2}+(M-1) Q_{M-1} P_{N-1}\right)\right],
\end{aligned}
$$


From the cubic equation

$$
\begin{aligned}
& z^{N-1}: \quad\left[-\frac{2}{3 \sqrt{3}} e_{-2}+\frac{2}{3} d_{-1}\left(2 b_{-1}+a_{-1}\right)\right]=\frac{4}{3} \epsilon N\left(2 b_{-1}+a_{-1}\right)^{2}+\epsilon N A_{-2}, \\
& z^{N-2}: \quad P_{N-1}\left[-\frac{2}{3 \sqrt{3}} e_{-2}+\frac{2}{3} d_{-1}\left(2 b_{-1}+a_{-1}\right)\right] \\
& +\left[-\frac{2}{3 \sqrt{3}} e_{-1}+\frac{2}{3} d_{0}\left(2 b_{-1}+a_{-1}\right)+\frac{2}{3} d_{-1}\left(2 b_{0}+a_{0}\right)-\frac{\epsilon}{2} d_{-1}\right] \\
& =2 \epsilon^{2} N(N-1)\left(2 b_{-1}+a_{-1}\right) \\
& +\frac{4}{3} \epsilon\left(2 N\left(2 b_{-1}+a_{-1}\right)\left(2 b_{0}+a_{0}\right)+P_{N-1}(N-1)\left(2 b_{-1}+a_{-1}\right)^{2}\right) \\
& +\epsilon\left(P_{N-1}(N-1) A_{-2}+N\left(A_{-1}-d_{-1}\right)\right) \\
& z^{N-3}: \quad P_{N-2}\left[-\frac{2}{3 \sqrt{3}} e_{-2}+\frac{2}{3} d_{-1}\left(2 b_{-1}+a_{-1}\right)\right] \\
& +P_{N-1}\left[-\frac{2}{3 \sqrt{3}} e_{-1}+\frac{2}{3} d_{0}\left(2 b_{-1}+a_{-1}\right)+\frac{2}{3} d_{-1}\left(2 b_{0}+a_{0}\right)-\frac{\epsilon}{2} d_{-1}\right] \\
& +\left[-\frac{2}{3 \sqrt{3}} e_{0}+\frac{2}{3} d_{0}\left(2 b_{0}+a_{0}\right)+\frac{2}{3} d_{-1}\left(2 b_{1}+a_{1}\right)-\epsilon d_{0}\right] \\
& =\epsilon^{3} N(N-1)(N-2) \\
& +2 \epsilon^{2}\left(N(N-2)\left(2 b_{0}+a_{0}\right)+P_{N-1}(N-1)(N-2)\left(2 b_{-1}+a_{-1}\right)\right) \\
& +\frac{4}{3} \epsilon\left(N\left(2 b_{0}+a_{0}\right)^{2}+2 P_{N-1}(N-1)\left(2 b_{-1}+a_{-1}\right)\left(2 b_{0}+a_{0}\right)\right. \\
& \left.+P_{N-2}(N-2)\left(2 b_{-1}+a_{-1}\right)^{2}\right) \\
& +\epsilon\left(P_{N-2}(N-2) A_{-2}+P_{N-1}(N-1)\left(A_{-1}-d_{-1}\right)+N\left(A_{0}-d_{0}\right)\right), \\
& z^{N-4}: \quad P_{N-3}\left[-\frac{2}{3 \sqrt{3}} e_{-2}+\frac{2}{3} d_{-1}\left(2 b_{-1}+a_{-1}\right)\right] \\
& +P_{N-2}\left[-\frac{2}{3 \sqrt{3}} e_{-1}+\frac{2}{3} d_{0}\left(2 b_{-1}+a_{-1}\right)+\frac{2}{3} d_{-1}\left(2 b_{0}+a_{0}\right)-\frac{\epsilon}{2} d_{-1}\right] \\
& +P_{N-1}\left[-\frac{2}{3 \sqrt{3}} e_{0}+\frac{2}{3} d_{0}\left(2 b_{0}+a_{0}\right)+\frac{2}{3} d_{-1}\left(2 b_{1}+a_{1}\right)-\epsilon d_{0}\right] \\
& +\left[-\frac{2}{3 \sqrt{3}} e_{1}+\frac{2}{3} d_{0}\left(2 b_{1}+a_{1}\right)\right] \\
& =\epsilon^{3} P_{N-1}(N-1)(N-2)(N-3) \\
& +2 \epsilon^{2}\left(N(N-3)\left(2 b_{1}+a_{1}\right)+P_{N-1}(N-1)(N-3)\left(2 b_{0}+a_{0}\right)\right. \\
& \left.+P_{N-2}(N-2)(N-3)\left(2 b_{-1}+a_{-1}\right)\right) \\
& +\frac{4}{3} \epsilon\left(2 N\left(2 b_{0}+a_{0}\right)\left(2 b_{1}+a_{1}\right)+P_{N-1}(N-1)\left(2 b_{0}+a_{0}\right)^{2}\right. \\
& \left.+2 P_{N-2}(N-2)\left(2 b_{-1}+a_{-1}\right)\left(2 b_{0}+a_{0}\right)+P_{N-3}(N-3)\left(2 b_{-1}+a_{-1}\right)^{2}\right) \\
& +\epsilon\left(P_{N-3}(N-3) A_{-2}+P_{N-2}(N-2)\left(A_{-1}-d_{-1}\right)\right. \\
& \left.+P_{N-1}(N-1)\left(A_{0}-d_{0}\right)+N A_{1}\right)
\end{aligned}
$$




$$
\begin{aligned}
& z^{N-5}: P_{N-4}\left[-\frac{2}{3 \sqrt{3}} e_{-2}+\frac{2}{3} d_{-1}\left(2 b_{-1}+a_{-1}\right)\right] \\
&+P_{N-3}\left[-\frac{2}{3 \sqrt{3}} e_{-1}+\frac{2}{3} d_{0}\left(2 b_{-1}+a_{-1}\right)+\frac{2}{3} d_{-1}\left(2 b_{0}+a_{0}\right)-\frac{\epsilon}{2} d_{-1}\right] \\
&+P_{N-2}\left[-\frac{2}{3 \sqrt{3}} e_{0}+\frac{2}{3} d_{0}\left(2 b_{0}+a_{0}\right)+\frac{2}{3} d_{-1}\left(2 b_{1}+a_{1}\right)-\epsilon d_{0}\right] \\
&+P_{N-1}\left[-\frac{2}{3 \sqrt{3}} e_{1}+\frac{2}{3} d_{0}\left(2 b_{1}+a_{1}\right)\right] \\
&=\epsilon^{3} P_{N-2}(N-2)(N-3)(N-4) \\
&+ \\
&+2 \epsilon^{2}\left(P_{N-1}(N-1)(N-4)\left(2 b_{1}+a_{1}\right)+P_{N-2}(N-2)(N-4)\left(2 b_{0}+a_{0}\right)\right. \\
&\left.+P_{N-3}(N-3)(N-4)\left(2 b_{-1}+a_{-1}\right)\right) \\
&+\frac{4}{3} \epsilon\left(2 P_{N-1}(N-1)\left(2 b_{0}+a_{0}\right)\left(2 b_{1}+a_{1}\right)+P_{N-2}(N-2)\left(2 b_{0}+a_{0}\right)^{2}\right. \\
&+\left.2 P_{N-3}(N-3)\left(2 b_{-1}+a_{-1}\right)\left(2 b_{0}+a_{0}\right)+P_{N-4}(N-4)\left(2 b_{-1}+a_{-1}\right)^{2}\right) \\
&+\epsilon\left(P_{N-4}(N-4) A_{-2}+P_{N-3}(N-3)\left(A_{-1}-d_{-1}\right)\right. \\
&\left.+P_{N-2}(N-2)\left(A_{0}-d_{0}\right)+P_{N-1}(N-1) A_{1}\right)+4 \epsilon N\left(b_{1}^{2}+a_{1} b_{1}\right),
\end{aligned}
$$

The corresponding equations of $Q_{M-k}$ can be obtained by setting $P_{N-k} \rightarrow Q_{M-k}, e_{k} \rightarrow$ $-e_{k}$ and $b_{k} \rightarrow a_{k}$. Again we can apply perturbation method.

To apply perturbation, we assume $\left|b_{1}\right| \ll\left|a_{1}\right|,\left|b_{1} b_{-1}\right| \ll 1$ and $\left|a_{1} a_{-1}\right| \ll 1$ so that $\left|P_{N-k}\right| \sim\left|b_{1}\right|^{k}$ is ensured. ${ }^{3}$ Then at the first order, we have from the quadratic equation:

$$
z^{N+M-2}: \quad d_{0}^{(1)}=\epsilon^{2}(N(N-1)-N M+M(M-1))+2 \epsilon\left[N b_{0}+M a_{0}\right],
$$

From the cubic equation:

$$
\begin{aligned}
z^{N-2}: \quad[ & \left.-\frac{2}{3 \sqrt{3}} e_{-1}^{(1)}+\frac{2}{3} d_{0}^{(1)}\left(2 b_{-1}+a_{-1}\right)+\frac{2}{3} d_{-1}\left(2 b_{0}+a_{0}\right)-\frac{\epsilon}{2} d_{-1}\right] \quad \text { (C.41) } \\
= & 2 \epsilon^{2} N(N-1)\left(2 b_{-1}+a_{-1}\right)+\frac{8}{3} \epsilon N\left(2 b_{-1}+a_{-1}\right)\left(2 b_{0}+a_{0}\right)+\epsilon N\left(A_{-1}-d_{-1}\right), \\
e_{-1}^{(1)}= & 4 \sqrt{3} \epsilon\left[M\left(a_{0} a_{-1}+a_{0} b_{-1}+b_{0} a_{-1}\right)-N\left(b_{0} b_{-1}+a_{0} b_{-1}+b_{0} a_{-1}\right)\right] \quad(\text { C. } 42) \\
& +\sqrt{3} \epsilon^{2}\left[a_{-1}\left(M^{2}-2 N^{2}+2 N M+2 N-\frac{5}{2} M\right)\right. \\
& \left.+b_{-1}\left(2 M^{2}-N^{2}-2 N M-2 M+\frac{5}{2} N\right)\right],
\end{aligned}
$$

\footnotetext{
${ }^{3}$ Although under this condition $Q_{M-k}$ cannot be found by perturbation, $d_{k}$ and $e_{k}$ can be totally fixed by symmetry. Notice that $d_{k}$ is invariant under the transformation $N \rightarrow M$ and $a_{l} \rightarrow b_{l}$, while $e_{k}$ is anti-invariant when $N \rightarrow M$ and $a_{l} \rightarrow b_{l}$. Thus the explicit dependence of $P_{N-k}$ is enough to determine $d_{k}$ and $e_{k}$.
} 


$$
\begin{array}{cl}
z^{N-3}: & {\left[-\frac{2}{3 \sqrt{3}} e_{0}^{(1)}+\frac{2}{3} d_{0}^{(1)}\left(2 b_{0}+a_{0}\right)-\epsilon d_{0}^{(1)}\right]} \\
& =\epsilon^{3} N(N-1)(N-2)+2 \epsilon^{2} N(N-2)\left(2 b_{0}+a_{0}\right)+\frac{4}{3} \epsilon N\left(2 b_{0}+a_{0}\right)^{2}+\epsilon N\left(A_{0}-d_{0}\right), \\
z^{N-4}: \quad & {\left[-\frac{2}{3 \sqrt{3}} e_{1}^{(1)}+\frac{2}{3} d_{0}^{(1)} a_{1}\right]=2 \epsilon^{2} N(N-3) a_{1}+4 \epsilon^{2} N a_{1}+4 \epsilon N b_{0} a_{1}, \quad \text { (C.44) }} \\
\left.e_{1}^{(1)}=a_{1}\left(2 \sqrt{3} \epsilon\left[M a_{0}-2 N b_{0}\right]+\sqrt{3} \epsilon^{2}[-2 N(N-1)-N M+M(M-1)]\right), \quad \text { (C. } 45\right) \\
z^{N-5:} \quad P_{N-1}^{(1)}\left[-\frac{2}{3 \sqrt{3}} e_{1}^{(1)}+\frac{2}{3} d_{0}^{(1)} a_{1}\right]-4 \epsilon N a_{1} b_{1} \\
=P_{N-1}^{(1)}\left(2 \epsilon^{2}(N-1)(N-4) a_{1}+\frac{8}{3} \epsilon(N-1)\left(2 b_{0}+a_{0}\right) a_{1}\right. \\
\left.\quad+\epsilon(N-1)\left(4 \epsilon a_{1}-\frac{4}{3}\left(2 a_{0} a_{1}+b_{0} a_{1}\right)\right)\right), \\
P_{N-1}^{(1)}=\frac{N b_{1}}{3 \epsilon(N-1)+b_{0}} .
\end{array}
$$

Second order contribution is given as follows:

$$
\begin{array}{rlrl}
z^{N+M-2}: d_{0}^{(2)}= & -2 \epsilon\left[b_{-1} P_{N-1}^{(1)}+a_{-1} Q_{M-1}^{(1)}\right], \\
d_{0}^{(2)}= & -2 \epsilon\left(\frac{N b_{1} b_{-1}}{3 \epsilon(N-1)+b_{0}}+\frac{M a_{1} a_{-1}}{3 \epsilon(M-1)+a_{0}}\right) . \\
\left.z^{N-2}: P_{N-1}\left[\frac{4}{3} \epsilon\left(2 b_{-1}+a_{-1}\right)^{2}+\epsilon N A_{-2}\right]+\left[-\frac{2}{3 \sqrt{3}} e_{-1}^{(2)}+\frac{2}{3} d_{0}^{(2)}\left(2 b_{-1}+a_{-1}\right)\right]=0, \quad \text { (C. } 50\right) \\
e_{-1}^{(2)}= & 2 \sqrt{3} \epsilon\left(\frac{\left(2 a_{-1}+b_{-1}\right) N}{3 \epsilon(N-1)+b_{0}} b_{1} b_{-1}-\frac{\left(a_{-1}+2 b_{-1}\right) M}{3 \epsilon(M-1)+a_{0}} a_{1} a_{-1}\right) . \\
z^{N-4}: \quad P_{N-1}^{(1)} & {\left[-\frac{2}{3 \sqrt{3}} e_{0}^{(1)}+\frac{2}{3} d_{0}^{(1)}\left(2 b_{0}+a_{0}\right)-\epsilon d_{0}^{(1)}\right]+\left[-\frac{2}{3 \sqrt{3}} e_{1}^{(2)}+\frac{4}{3} d_{0}^{(1)} b_{1}\right]} \\
= & \epsilon^{3} P_{N-1}^{(1)}(N-1)(N-2)(N-3) \\
& +2 \epsilon^{2}\left(N(N-3) 2 b_{1}+P_{N-1}^{(1)}(N-1)(N-3)\left(2 b_{0}+a_{0}\right)\right) \\
& +\frac{4}{3} \epsilon\left(4 N\left(2 b_{0}+a_{0}\right) b_{1}+P_{N-1}^{(1)}(N-1)\left(2 b_{0}+a_{0}\right)^{2}\right) \\
& +\epsilon\left(P_{N-1}^{(1)}(N-1)\left(A_{0}-d_{0}\right)+N\left[4 \epsilon b_{1}-\frac{4}{3}\left(2 b_{0}+a_{0}\right) b_{1}\right]\right), \\
e_{1}^{(2)} & 2 \sqrt{3} b_{1}\left(\epsilon\left[2 M a_{0}-3 N a_{0}-4 N b_{0}\right]+\epsilon^{2}\left[-2 N^{2}+5 N-N M+M(M-1)\right]\right) \\
& +\frac{3 \sqrt{3}}{2} \frac{N b_{1}}{3 \epsilon(N-1)+b_{0}}\left(4 \epsilon\left(a_{0}+b_{0}\right) b_{0}+2 \epsilon^{2}\left[b_{0}(3 N-5)+a_{0}(2 N-2-M)\right]\right. \\
& \left.+\epsilon^{3}[2(N-1)(N-3)-N M+M(M-1)]\right) .
\end{array}
$$


From this consideration we have

$$
\begin{aligned}
d_{0} & =d_{0}^{(1)}+d_{0}^{(2)}+\ldots=D_{0}+D_{1} b_{-1} b_{1}+D_{2} a_{-1} a_{1}+\text { higher order } \\
e_{1} & =e_{1}^{(1)}+e_{1}^{(2)}+\ldots=D_{3} a_{1}+D_{4} b_{1}+\text { higher order } \\
e_{-1} & =e_{-1}^{(1)}+e_{-1}^{(2)}+\ldots \\
& =D_{5} b_{-1}+D_{6} a_{-1}+D_{7}\left(2 a_{-1}+b_{-1}\right) b_{1} b_{-1}+D_{8}\left(a_{-1}+2 b_{-1}\right) a_{1} a_{-1}+\text { higher order }
\end{aligned}
$$

where all $D_{k}$ are functions of $b_{0}, a_{0}$, which can be read off from the above equations. We compare these results with the $\epsilon$ expansion (3.31)-(3.33) and find they agree with each other, by calculating (3.31)-(3.33) further to order $\mathcal{O}\left(\eta_{0}^{2}\right)$ with $M=M_{1}$ and $N=N_{1}$ :

$$
\begin{aligned}
d_{0}^{(1 ; 1)}= & 2 \epsilon\left(b_{0} N+a_{0} M\right)-2 \epsilon\left(\frac{M}{a_{0}} a_{1} a_{-1}+\frac{N}{b_{0}} b_{1} b_{-1}\right)+\mathcal{O}\left(\eta_{0}^{2}\right), \\
e_{1}^{(1 ; 1)}= & 2 \sqrt{3} \epsilon\left[a_{0}\left(a_{1}+2 b_{1}\right) M-b_{0}\left(2 a_{1}+b_{1}\right) N\right] \\
& -2 \sqrt{3} \epsilon\left(\frac{\left(a_{1}+2 b_{1}\right) M}{a_{0}} a_{1} a_{-1}-\frac{\left(2 a_{1}+b_{1}\right) N}{b_{0}} b_{1} b_{-1}\right)+\mathcal{O}\left(\eta_{0}^{2}\right), \\
e_{-1}^{(1 ; 1)}= & 4 \sqrt{3} \epsilon\left[a_{0}\left(a_{-1} M+b_{-1}(M-N)\right)+b_{0}\left(a_{-1}(M-N)-b_{-1} N\right)\right] \\
& -2 \sqrt{3} \epsilon\left(\frac{\left(a_{-1}+2 b_{-1}\right) M}{a_{0}} a_{1} a_{-1}-\frac{\left(2 a_{-1}+b_{-1}\right) N}{b_{0}} b_{1} b_{-1}\right)+\mathcal{O}\left(\eta_{0}^{2}\right) .
\end{aligned}
$$

Open Access. This article is distributed under the terms of the Creative Commons Attribution License (CC-BY 4.0), which permits any use, distribution and reproduction in any medium, provided the original author(s) and source are credited.

\section{References}

[1] P.C. Argyres and M.R. Douglas, New phenomena in SU(3) supersymmetric gauge theory, Nucl. Phys. B 448 (1995) 93 [hep-th/9505062] [INSPIRE].

[2] L.F. Alday, D. Gaiotto and Y. Tachikawa, Liouville Correlation Functions from Four-dimensional Gauge Theories, Lett. Math. Phys. 91 (2010) 167 [arXiv:0906.3219] [INSPIRE].

[3] N.A. Nekrasov, Seiberg-Witten prepotential from instanton counting, Adv. Theor. Math. Phys. 7 (2003) 831 [hep-th/0206161] [InSPIRE].

[4] N.A. Nekrasov, Seiberg-Witten prepotential from instanton counting, hep-th/0306211 [INSPIRE].

[5] N. Nekrasov and A. Okounkov, Seiberg-Witten theory and random partitions, Prog. Math. 244 (2006) 525 [hep-th/0306238] [INSPIRE].

[6] D. Gaiotto, G.W. Moore and A. Neitzke, Wall-crossing, Hitchin Systems and the WKB Approximation, arXiv:0907.3987 [INSPIRE].

[7] G. Bonelli and A. Tanzini, Hitchin systems, $N=2$ gauge theories and $W$-gravity, Phys. Lett. B 691 (2010) 111 [arXiv:0909.4031] [INSPIRE].

[8] D. Nanopoulos and D. Xie, Hitchin Equation, Singularity and $N=2$ Superconformal Field Theories, JHEP 03 (2010) 043 [arXiv:0911.1990] [INSPIRE]. 
[9] N.J. Hitchin, Stable bundles and integrable systems, Duke Math. J. 54 (1987) 91 [INSPIRE].

[10] R. Donagi and E. Witten, Supersymmetric Yang-Mills theory and integrable systems, Nucl. Phys. B 460 (1996) 299 [hep-th/9510101] [INSPIRE].

[11] S. Cecotti, A. Neitzke and C. Vafa, R-Twisting and $4 d / 2 d$ Correspondences, arXiv:1006.3435 [INSPIRE].

[12] S. Cecotti and C. Vafa, Classification of complete $N=2$ supersymmetric theories in 4 dimensions, Surveys in differential geometry 18 (2013) [arXiv:1103.5832] [INSPIRE].

[13] T. Eguchi and K. Maruyoshi, Penner Type Matrix Model and Seiberg-Witten Theory, JHEP 02 (2010) 022 [arXiv:0911.4797] [INSPIRE].

[14] D. Gaiotto and J. Teschner, Irregular singularities in Liouville theory and Argyres-Douglas type gauge theories, I, JHEP 12 (2012) 050 [arXiv: 1203.1052] [INSPIRE].

[15] D. Gaiotto, Asymptotically free $\mathcal{N}=2$ theories and irregular conformal blocks, J. Phys. Conf. Ser. 462 (2013) 012014 [arXiv:0908.0307] [InSPIRE].

[16] E. Felinska, Z. Jaskolski and M. Kosztolowicz, Whittaker pairs for the Virasoro algebra and the Gaiotto-BMT states, J. Math. Phys. 53 (2012) 033504 [Erratum ibid. 53 (2012) 129902] [arXiv:1112.4453] [INSPIRE].

[17] R.C. Penner, Perturbative series and the moduli space of Riemann surfaces, J. Diff. Geom. 27 (1988) 35 [inSPIRE].

[18] R. Dijkgraaf and C. Vafa, Toda Theories, Matrix Models, Topological Strings and $N=2$ Gauge Systems, arXiv:0909.2453 [INSPIRE].

[19] H. Itoyama and T. Oota, Method of Generating q-Expansion Coefficients for Conformal Block and $N=2$ Nekrasov Function by beta-Deformed Matrix Model, Nucl. Phys. B 838 (2010) 298 [arXiv:1003.2929] [INSPIRE].

[20] S.K. Choi, C. Rim and H. Zhang, Virasoro irregular conformal block and beta deformed random matrix model, Phys. Lett. B $\mathbf{7 4 2}$ (2015) 50 [arXiv:1411.4453] [INSPIRE].

[21] N.A. Nekrasov and S.L. Shatashvili, Quantization of Integrable Systems and Four Dimensional Gauge Theories, arXiv:0908.4052 [INSPIRE].

[22] T. Nishinaka and C. Rim, Matrix models for irregular conformal blocks and Argyres-Douglas theories, JHEP 10 (2012) 138 [arXiv:1207.4480] [INSPIRE].

[23] A. Marshakov, A. Mironov and A. Morozov, On AGT Relations with Surface Operator Insertion and Stationary Limit of Beta-Ensembles, J. Geom. Phys. 61 (2011) 1203 [arXiv: 1011.4491] [INSPIRE].

[24] S.-K. Choi and C. Rim, Parametric dependence of irregular conformal block, JHEP 04 (2014) 106 [arXiv:1312.5535] [INSPIRE].

[25] L. Chekhov, Logarithmic potential $\beta$-ensembles and Feynman graphs, arXiv:1009.5940 [INSPIRE].

[26] L.O. Chekhov, B. Eynard and O. Marchal, Topological expansion of $\beta$-ensemble model and quantum algebraic geometry in the sectorwise approach, Theor. Math. Phys. 166 (2011) 141 [arXiv: 1009.6007] [INSPIRE].

[27] R. Dijkgraaf and C. Vafa, On geometry and matrix models, Nucl. Phys. B 644 (2002) 21 [hep-th/0207106] [INSPIRE]. 
[28] G. Bonelli, K. Maruyoshi and A. Tanzini, Quantum Hitchin Systems via beta-deformed Matrix Models, arXiv:1104.4016 [INSPIRE].

[29] C. Rim and H. Zhang, Classical Virasoro irregular conformal block II, JHEP 09 (2015) 097 [arXiv: 1506.03561] [inSPIRE].

[30] H. Kanno, K. Maruyoshi, S. Shiba and M. Taki, $W_{3}$ irregular states and isolated $N=2$ superconformal field theories, JHEP 03 (2013) 147 [arXiv: 1301.0721] [INSPIRE].

[31] A. Mironov, A. Morozov and S. Shakirov, A direct proof of AGT conjecture at $\beta=1$, JHEP 02 (2011) 067 [arXiv:1012.3137] [INSPIRE].

[32] H. Zhang and Y. Matsuo, Selberg Integral and SU(N) AGT Conjecture, JHEP 12 (2011) 106 [arXiv:1110.5255] [INSPIRE].

[33] Y. Matsuo, C. Rim and H. Zhang, Construction of Gaiotto states with fundamental multiplets through Degenerate DAHA, JHEP 09 (2014) 028 [arXiv:1405.3141] [INSPIRE].

[34] J.-E. Bourgine, Spherical Hecke algebra in the Nekrasov-Shatashvili limit, JHEP 01 (2015) 114 [arXiv:1407.8341] [INSPIRE].

[35] B. Eynard, Topological expansion for the 1-Hermitian matrix model correlation functions, JHEP 11 (2004) 031 [hep-th/0407261] [INSPIRE].

[36] S.K. Choi and C. Rim, Irregular matrix model with $\mathcal{W}$ symmetry, J. Phys. A 49 (2016) 075201 [arXiv: 1506.02421] [INSPIRE].

[37] G. Bonelli, K. Maruyoshi and A. Tanzini, Wild Quiver Gauge Theories, JHEP 02 (2012) 031 [arXiv:1112.1691] [INSPIRE].

[38] A.B. Zamolodchikov and A.B. Zamolodchikov, Structure constants and conformal bootstrap in Liouville field theory, Nucl. Phys. B 477 (1996) 577 [hep-th/9506136] [INSPIRE].

[39] V.A. Fateev and A.V. Litvinov, Correlation functions in conformal Toda field theory. I., JHEP 11 (2007) 002 [arXiv:0709.3806] [INSPIRE].

[40] C. Rim and H. Zhang, Classical Virasoro irregular conformal block, JHEP 07 (2015) 163 [arXiv: 1504.07910] [INSPIRE].

[41] N. Wyllard, $A(N-1)$ conformal Toda field theory correlation functions from conformal $\mathcal{N}=2 \mathrm{SU}(N)$ quiver gauge theories, JHEP 11 (2009) 002 [arXiv:0907.2189] [INSPIRE].

[42] S. Kanno, Y. Matsuo, S. Shiba and Y. Tachikawa, $N=2$ gauge theories and degenerate fields of Toda theory, Phys. Rev. D 81 (2010) 046004 [arXiv:0911.4787] [INSPIRE].

[43] V.A. Alba, V.A. Fateev, A.V. Litvinov and G.M. Tarnopolskiy, On combinatorial expansion of the conformal blocks arising from AGT conjecture, Lett. Math. Phys. 98 (2011) 33 [arXiv: 1012.1312] [INSPIRE].

[44] H. Itoyama, K. Maruyoshi and T. Oota, The Quiver Matrix Model and 2d-4d Conformal Connection, Prog. Theor. Phys. 123 (2010) 957 [arXiv:0911.4244] [INSPIRE].

[45] E. Witten, Solutions of four-dimensional field theories via M-theory, Nucl. Phys. B 500 (1997) 3 [hep-th/9703166] [INSPIRE].

[46] D. Gaiotto, $N=2$ dualities, JHEP 08 (2012) 034 [arXiv:0904.2715] [INSPIRE].

[47] T. Eguchi and K. Maruyoshi, Seiberg-Witten theory, matrix model and AGT relation, JHEP 07 (2010) 081 [arXiv: 1006.0828] [INSPIRE]. 
[48] T. Nishinaka and C. Rim, $\beta$-Deformed Matrix Model and Nekrasov Partition Function, JHEP 02 (2012) 114 [arXiv:1112.3545] [INSPIRE].

[49] D. Xie, General Argyres-Douglas Theory, JHEP 01 (2013) 100 [arXiv:1204.2270] [INSPIRE].

[50] A. Litvinov, S. Lukyanov, N. Nekrasov and A. Zamolodchikov, Classical Conformal Blocks and Painleve VI, JHEP 07 (2014) 144 [arXiv: 1309.4700] [INSPIRE].

[51] S. Kanno, Y. Matsuo and H. Zhang, Extended Conformal Symmetry and Recursion Formulae for Nekrasov Partition Function, JHEP 08 (2013) 028 [arXiv:1306.1523] [INSPIRE].

[52] A. Klemm, K. Landsteiner, C.I. Lazaroiu and I. Runkel, Constructing gauge theory geometries from matrix models, JHEP 05 (2003) 066 [hep-th/0303032] [INSPIRE].

[53] S.G. Naculich, H.J. Schnitzer and N. Wyllard, Cubic curves from matrix models and generalized Konishi anomalies, JHEP 08 (2003) 021 [hep-th/0303268] [INSPIRE].

[54] R. Schiappa and N. Wyllard, An Ar threesome: Matrix models, $2 d$ CFTs and $4 d N=2$ gauge theories, J. Math. Phys. 51 (2010) 082304 [arXiv:0911.5337] [InSPIRE].

[55] V.A. Fateev and A.B. Zamolodchikov, Conformal Quantum Field Theory Models in Two-Dimensions Having $Z_{3}$ Symmetry, Nucl. Phys. B 280 (1987) 644 [INSPIRE]. 